\title{
Stability properties of an inverse parabolic problem with unknown boundaries
}

\author{
Michele Di Cristo $†$ Luca Rondi ${ }^{\dagger}$ and Sergio Vessella ${ }^{\ddagger}$
}

\begin{abstract}
We treat the stability issue for an inverse problem arising from nondestructive evaluation by thermal imaging. We consider the determination of an unknown portion of the boundary of a thermic conducting body by overdetermined boundary data for a parabolic initial-boundary value problem. We prove a stability estimate with a single measurement with some a priori information on the unknown part of the boundary and minimal assumptions on the data, in particular on the thermal conductivity. Then, we obtain that even when the unknown part of the boundary is a priori known to be smooth, the data are as regular as possible and all possible measurements are taken into account, still the problem is exponentially ill-posed. Therefore, our stability estimate is optimal.
\end{abstract}

AMS 2000 Mathematics Subject Classification Primary 35R30. Secondary 35B60, 33C90.

Keywords inverse problems, stability, parabolic equations, unique continuation, Bessel functions.

\section{Introduction}

Let $\Omega$ be a bounded domain in $\mathbb{R}^{N}, N \geq 2$, with a sufficiently smooth boundary $\partial \Omega$, a closed part of which, say $I$, is not known and not accessible. For instance, $I$ could be some interior component of $\partial \Omega$ or some inaccessible portion of the exterior component of $\partial \Omega$. On the other hand, we assume that the set $A=\partial \Omega \backslash I$ is accessible and known. Let $T$ be a positive number and let $\kappa=\kappa(x, t),(x, t) \in$ $\mathbb{R}^{N} \times \mathbb{R}$, be a symmetric $N \times N$ matrix whose entries are Lipschitz continuous (real) functions. We assume that $\kappa$ is also uniformly elliptic. Given a nontrivial function $f$ on $A \times[0, T]$ such that $\operatorname{supp} f$ is compactly contained in $A \times[0, T]$, let us consider the initial-boundary value problem

$$
\begin{cases}\partial_{t} u-\operatorname{div}(\kappa(x, t) \nabla u)=0 & \text { in } \Omega \times(0, T), \\ u(x, 0)=0 & x \in \bar{\Omega}, \\ u=0 & \text { on } I \times[0, T] \\ u=f & \text { on } A \times[0, T] .\end{cases}
$$

${ }^{\dagger}$ Dipartimento di Scienze Matematiche, Università degli Studi di Trieste, Italy. E-mail: M. Di Cristo dicristo@dsm.univ.trieste.it and L. Rondi rondi@dsm. univ.trieste.it

¥Dipartimento di Matematica per le Decisioni, Università degli Studi di Firenze, Italy. E-mail: sergio.vessella@dmd.unifi.it 
Given an open portion $\Sigma$ of $\partial \Omega$ such that $\Sigma \subset A$, we consider the inverse problem of determining $I$ from the knowledge of $\kappa \nabla u \cdot \nu$ on $\Sigma \times[0, T]$, where $\nu$ denotes the exterior normal to $\Omega$.

This problem arises from nondestructive testing using thermal imaging. In fact, $\Omega$ represents a thermic conducting body, with thermal conductivity $\kappa$, $A$ is a known part of the boundary and $I$ represents an unknown privileged isothermal surface, such as a solidification front, [2], a corroded portion of $\partial \Omega$ or the boundary of a cavity inside $\Omega,[3,20]$. We prescribe the temperature on the accessible part of the boundary, $A$, and we measure on $\Sigma \times[0, T]$ the heat flux exiting the body, and, through this additional measurement, we want to determine $I$.

The uniqueness result for the problem above has been proved by Isakov, see [11]. In [5] it is shown via a counterexample that uniqueness may fail when $u(\cdot, 0) \not \equiv 0$.

In this paper we approach the stability issue of this problem from two different points of view. First of all, we prove a stability estimate under some a priori information on $I$ and some assumptions on $A, \kappa$ and the oscillation character of $f$. The stability estimate we prove is of logarithmic type and it is therefore very weak, however it is essentially optimal. In fact, we then analyse the instability character of the problem and we find that this inverse problem is exponentially unstable, that is logarithmic stability estimates are best possible. We wish to remark that, for what concerns the stability estimate, the aim is to keep as minimal as possible the a priori information on the unknown $I$ and the assumptions on the data $A, \kappa$ and $f$ while still keeping the optimality of the estimate. Conversely, to properly analyse the instability of the problem, we study what happens in the most favourable situation, that is we suppose to have strong a priori information on $I, A$ and $\kappa$ as simple as possible and, more notably, instead of a single measurement with a given $f$ we take into account all possible measurements. Still the instability is of exponential type, and this means that performing different or more measurements does not substantially improve the stability of the problem.

By the stability estimates and the instability analysis we can therefore characterize in a quite precise manner the modulus of continuity of the map which associates to the measurement $\kappa \nabla u \cdot \nu$ on $\Sigma \times[0, T]$ the unknown part of the boundary $I$.

More precisely, we wish to remark that the stability estimate is established when $I$ is assumed to be $C^{1, \beta}, 0<\beta \leq 1$, whereas $A$ is just Lipschitz. Furthermore, $\kappa$ may depend on both time and space variables. We notice that in [5] an analogous logarithmic stability estimate has been proved when $A$ is assumed to be $C^{1,1}$ and $\kappa$ does not depend on $t$.

The proof of the stability estimate has the same structure of those given in $[1,4,5,18]$. As in these papers, the main effort consists of deducing some quantitative estimates of unique continuation, in particular three cylinder inequalities in the interior and at the boundary and stability estimates for Cauchy problems. An optimal three cylinder inequality in the interior has been proved in $[9,21]$ and it is recalled in Theorem 3.4. We also need an optimal three cylinder inequality at the boundary and this is obtained in Theorem 3.5. The main novelty here is the dependence of the thermal conductivity by time, too. Let us also note that the stability estimate for Cauchy problems, Theorem 3.6, has been obtained for a Lipschitz boundary. 
In turn, in order to obtain such quantitative estimates of unique continuation, the main effort is to find a suitable Carleman estimate, see Theorem 3.1.

Instead, in the instability analysis we deal with the following framework. We assume that $\Omega=B_{1}(0) \backslash D$ where $D$ represents an unknown cavity. Therefore we identify $A$ with the exterior boundary of $\Omega, \partial B_{1}(0)$, and $I$ with the boundary of the cavity, $\partial D$. We further assume that $\kappa$ is identically equal to the identity matrix, that is the body is homogeneous and isotropic. Let us fix an integer $m$. To each $C^{m}$ regular cavity $D$ we associate its Dirichlet-to-Neumann map $\mathcal{D}(D)$, that is the operator that maps each prescribed temperature $f$ on $A \times[0, T]$ into the corresponding heat flux $\left.\frac{\partial u}{\partial \nu}\right|_{A \times[0, T]}$, where $u$ solves (1.1). We establish the instability properties of the function that associates to each operator $\mathcal{D}(D)$ the corresponding cavity $D$.

The basic idea of the method we use for such a purpose goes back to [12]. More recently, this idea has been applied successfully to the inverse problem of conductivity, see [17]. Later, in [7], the method has been formulated in an abstract framework suitable to be applied in the context of inverse problems. In that paper, in fact, the abstract formulation has been applied to many different inverse elliptic problems. Here we also make use of the abstract setting, however the parabolic case presents additional difficulties with respect to the elliptic case. A crucial step in order to apply the abstract method relies on the construction of a sequence $\left\{u_{k}\right\}_{k \in \mathbb{N}}$ of solutions to an auxiliary initial-boundary value problem (namely (1.1) with $\Omega=B_{1}(0)$, that is $D=\emptyset$ ), satisfying the following properties. First, the linear space generated by $\left\{u_{k}\right\}_{k \in \mathbb{N}}$ should be dense among all possible solutions to the auxiliary problem and, second, $u_{k}$ should decay exponentially with respect to $k$ on any compact subset of $B_{1}(0)$. In the elliptic case, such a sequence is provided by the harmonic polynomials, whereas in the parabolic case its construction is much more delicate and is performed in Section 5, with the crucial exponential decay estimate given in Proposition 5.5.

The plan of the paper is as follows. In Section 2 we state the main results of the paper: the stability estimate, Theorem 2.4 , and the exponential instability, Theorem 2.5. In Section 3 we prove the Carleman inequality, Theorem 3.1, and obtain the quantitative estimates of unique continuation. Then, the proof of Theorem 2.4 is concluded in Section 4. Finally, in Section 5, we prove Theorem 2.5 .

\section{Acknowledgements}

The authors wish to thank Luis Escauriaza for useful discussions on the subject of this work and acknowledge the support by MIUR under grant n. 2002013279.

\section{Statement of the main results}

We begin by giving some notation and definitions. We shall fix the space dimension $N, N \geq 2$, throughout the paper. We shall use the letter $C$ or $K$ to denote positive constants. The value of the constants may change from line to line, but we shall specify their dependence everywhere they appear (sometimes we emphasize their difference writing $\left.C_{0}, C_{1}, \ldots\right)$. We shall always omit the dependence of the constants on $N$.

For every $x \in \mathbb{R}^{N}$ we shall set $x=\left(x^{\prime}, x_{N}\right)$, where $x^{\prime} \in \mathbb{R}^{N-1}$ and $x_{N} \in \mathbb{R}$, and we shall denote by $B_{r}(x)$ and $B_{r}^{\prime}\left(x^{\prime}\right)$, respectively, the open ball in $\mathbb{R}^{N}$ 
centred at $x$ of radius $r$ and the open ball in $\mathbb{R}^{N-1}$ centred at $x^{\prime}$ of radius $r$. Sometimes we shall write $B_{r}$ and $B_{r}^{\prime}$ instead of $B_{r}(0)$ and $B_{r}^{\prime}(0)$, respectively.

Let $r, t_{0}$ be positive numbers. For every $x \in \mathbb{R}^{N}, \zeta \in \mathbb{R}^{N}$ such that $|\zeta|=1$ and $\alpha \in(0, \pi)$ we shall denote by $C(x, \zeta, \alpha, r)$ the cone $\left\{y \in B_{r}(x): \frac{(y-x) \cdot \zeta}{|y-x|}>\right.$ $\cos \alpha\}$ and we shall denote by $C^{t_{0}}(x, \zeta, \alpha, r)$ the set $C(x, \zeta, \alpha, r) \times\left(0, t_{0}\right)$. We shall denote by $D_{r}^{t_{0}}(x)$ the cylinder $B_{r}(x) \times\left(0, t_{0}\right)$. For every function $\psi$ on $\mathbb{R}^{N-1}$ such that $\psi(0)=0$, we shall denote by $D_{\psi, r}^{t_{0}}$ the set $\left\{\left(x^{\prime}, x_{N}, t\right) \in B_{r} \times\left(0, t_{0}\right)\right.$ : $\left.\psi\left(x^{\prime}\right)<x_{N}\right\}$. When dealing with $N+1$ variables $(x, t)$, with $x=\left(x_{1}, \ldots, x_{N}\right)$, we shall denote $\nabla=\nabla_{x}$, div $=\operatorname{div}_{x}, \Delta=\operatorname{div}(\nabla), D^{2}=D_{x}^{2}$. We shall write, for brevity, $\partial_{i} f=\frac{\partial f}{\partial x_{i}}, \partial_{t} f=\frac{\partial f}{\partial t}$. Finally, we shall denote by $\mathcal{I}$ the identity matrix.

Definition 2.1 Let $\Omega$ be a bounded domain in $\mathbb{R}^{N}$. Given a nonnegative integer $m$ and $\beta, 0 \leq \beta \leq 1$, we shall say that a portion $\Sigma$ of $\partial \Omega$ is of $C^{m, \beta}$ class with constants $R_{0}, E>0$, if for every $P \in \Sigma$, there exists a rigid transformation of coordinates under which we have $P=0$ and

$$
\Omega \cap B_{R_{0}}=\left\{\left(x^{\prime}, x_{N}\right) \in B_{R_{0}}: x_{N}>\varphi\left(x^{\prime}\right)\right\},
$$

where $\varphi$ is a $C^{m, \beta}$ function on $B_{R_{0}}^{\prime}$ satisfying $\varphi(0)=0,\|\varphi\|_{C^{m, \beta}\left(B_{R_{0}}^{\prime}\right)} \leq E R_{0}$ and, whenever $m \geq 1$, also $|\nabla \varphi(0)|=0$. We remark that when $m=0$ and $\beta=1$ we shall also speak of Lipschitz class and that when $\beta=0$ we shall speak of $C^{m}$ class.

Remark 2.2 We have chosen to normalize all norms in such a way that their terms are dimensionally homogeneous and coincide with the standard definition when the dimensional parameter $R_{0}$ is equal to one. For instance, we have that

$$
\|\varphi\|_{C^{1, \beta}\left(B_{R_{0}}^{\prime}\right)}=\|\varphi\|_{L^{\infty}\left(B_{R_{0}}^{\prime}\right)}+R_{0}\|\nabla \varphi\|_{L^{\infty}\left(B_{R_{0}}^{\prime}\right)}+R_{0}^{1+\beta}|\nabla \varphi|_{\beta, B_{R_{0}}^{\prime}},
$$

where

$$
|\nabla \varphi|_{\beta, B_{R_{0}}^{\prime}}=\sup _{\substack{x^{\prime}, y^{\prime} \in B_{R_{0}^{\prime}}^{\prime} \\ x^{\prime} \neq y^{\prime}}} \frac{\left|\nabla \varphi\left(x^{\prime}\right)-\nabla \varphi\left(y^{\prime}\right)\right|}{\left|x^{\prime}-y^{\prime}\right|^{\beta}} .
$$

In the same fashion, if $D=\Omega \times(0, T), T>0$, and $u$ is a function belonging to $H^{2,1}(D)$ the norm $\|u\|_{H^{2,1}(D)}$ is meant as follows

$$
\|u\|_{H^{2,1}(D)}^{2}=\frac{1}{T R_{0}^{N}} \int_{D}\left(u^{2}+R_{0}^{2}|\nabla u|^{2}+R_{0}^{4}\left|D^{2} u\right|^{2}+T^{2}\left(\partial_{t} u\right)^{2}\right) \mathrm{d} x \mathrm{~d} t,
$$

and so on for any other integral or fractional order Sobolev space defined on $D$ or on $\Sigma \times(0, T), \Sigma$ being a portion of $\partial \Omega$. For what concerns the definition and main properties of these Sobolev spaces we refer to [16].

\subsection{The stability result}

Let $M, R_{0}, E, F, \lambda, \Lambda, T$ and $\beta$ be given positive numbers with $\lambda, \beta \in(0,1]$. Theorem 2.4 below is based on the following assumptions and a priori information.

Assumptions on the domain and the accessible part of the boundary 
We assume that $\Omega$ is a bounded domain in $\mathbb{R}^{N}$ satisfying

$$
|\Omega| \leq M R_{0}^{N},
$$

where $|\Omega|$ denotes the Lebesgue measure of $\Omega$. We also assume that

$$
\partial \Omega \text { is of } C^{0,1} \text { class with constants } R_{0}, E \text {. }
$$

We shall distinguish two nonempty parts $A, I$ of $\partial \Omega$ which satisfy

$$
I \cup A=\partial \Omega, \quad \stackrel{\circ}{I} \cap \stackrel{\circ}{A}=\emptyset, \quad I \cap A=\partial A=\partial I,
$$

where interiors and boundaries are intended here in the relative topology of $\partial \Omega$.

Moreover we assume that we can select a portion $\Sigma$ of $A$ satisfying

$$
\partial \Omega \cap B_{R_{0}}\left(P_{1}\right) \subset \Sigma \subset A_{R_{0}},
$$

where $P_{1}$ is a point belonging to $\Sigma$ and $A_{R_{0}}=\left\{x \in \partial \Omega\right.$ : $\left.\operatorname{dist}(x, I) \geq R_{0}\right\}$.

Remark 2.3 Observe that condition (2.2) above implies a lower bound on the diameter of $\Omega$ and on the diameter of every connected component of $\partial \Omega$. On the other hand, conditions (2.1) and (2.2) imply an upper bound on the diameter of $\Omega$.

A priori information on the unknown part of the boundary We suppose that

$$
I \text { is of } C^{1, \beta} \text { class with constants } R_{0}, E \text {. }
$$

\section{Assumption about the boundary data}

We shall assume that the Dirichlet data $f$ appearing in problem (1.1) belongs to $H^{1 / 2,1 / 4}(A \times(0, T))$, it is nontrivial and satisfies

$$
\operatorname{supp} f \subset A_{R_{0}} \times[0, T], \quad \frac{\|f\|_{H^{1 / 2,1 / 4}(A \times(0, T))}}{\|f\|_{L^{2}(A \times(0, T))}} \leq F .
$$

Let us notice that $f$ can be trivially extended to $\partial \Omega \times(0, T)$ by setting $f=0$ on $I \times(0, T)$. In such a way, denoting

$$
S_{T}=\partial \Omega \times(0, T),
$$

we shall often consider $f$ as belonging to $H^{1 / 2,1 / 4}\left(S_{T}\right)$ and we shall set, unless otherwise specified,

$$
\|f\|=\|f\|_{H^{1 / 2,1 / 4}\left(S_{T}\right)} .
$$

Assumption about the thermal conductivity

We assume that the thermal conductivity $\kappa=\kappa(x, t),(x, t) \in \mathbb{R}^{N} \times \mathbb{R}$, is a symmetric $N \times N$ matrix satisfying the following conditions for every $(x, t)$, $(y, s) \in \mathbb{R}^{N+1}$ and every $\xi \in \mathbb{R}^{N}$

$$
\begin{aligned}
& \lambda|\xi|^{2} \leq \kappa(x, t) \xi \cdot \xi \leq \lambda^{-1}|\xi|^{2}, \\
& |\kappa(x, t)-\kappa(y, s)| \leq \Lambda\left(\frac{|x-y|}{R_{0}}+\frac{|t-s|}{T}\right) .
\end{aligned}
$$


In the sequel we shall refer to the set of numbers $\left\{R_{0}^{2} / T, M, E, F, \lambda, \Lambda, \beta\right\}$ as to the a priori data. We shall also use the following notation

$$
W(\Omega \times(0, T))=\left\{v: v \in L^{2}\left((0, T) ; H^{1}(\Omega)\right), \partial_{t} v \in L^{2}\left((0, T) ; H^{-1}(\Omega)\right)\right\} .
$$

Theorem 2.4 Let $\Omega_{1}, \Omega_{2}$ be two domains satisfying (2.1), (2.2). For any $i=$ 1,2 , let $A_{i}, I_{i}$, satisfying $(2.3)$, be the accessibile and inaccessible part of $\partial \Omega_{i}$, respectively. Assume that $A_{1}=A_{2}=A$ and that $\Omega_{1}$ and $\Omega_{2}$ lie on the same side of $A$. Let us take $\Sigma \subset A$ satisfying (2.4). Finally, we suppose that, for any $i=1,2, I_{i}$ satisfies the a priori information (2.5).

Let us assume that (2.6), (2.7) and (2.8) are also satisfied and let $u_{i} \in$ $W\left(\Omega_{i} \times(0, T)\right)$ be the weak solution to (1.1) when $\Omega=\Omega_{i}, i=1,2$. If, given $\varepsilon>0$, we have

$$
R_{0}^{1 / 2}\left\|\kappa \nabla u_{1} \cdot \nu-\kappa \nabla u_{2} \cdot \nu\right\|_{L^{2}(\Sigma \times(0, T))} \leq \varepsilon,
$$

then we have

$$
d_{\mathcal{H}}\left(\bar{\Omega}_{1}, \bar{\Omega}_{2}\right) \leq R_{0} \omega(\varepsilon /\|f\|),
$$

where $\omega$ is an increasing continuous function on $[0, \infty)$ which satisfies

$$
\omega(s) \leq C|\log s|^{-C_{1}}, \quad \text { for every } s<1,
$$

and $C, C_{1}$ are positive constants depending on the a priori data only.

Here $d_{\mathcal{H}}$ denotes the Hausdorff distance, namely

$$
d_{\mathcal{H}}\left(\bar{\Omega}_{1}, \bar{\Omega}_{2}\right)=\max \left\{\sup _{x \in \bar{\Omega}_{1}} \operatorname{dist}\left(x, \bar{\Omega}_{2}\right), \sup _{x \in \bar{\Omega}_{2}} \operatorname{dist}\left(x, \bar{\Omega}_{1}\right)\right\} .
$$

\subsection{The instability result}

Let $\Omega=B_{1} \backslash D$, where $D$ is a compact subset of $B_{1}$. Let $A=\partial B_{1}=\mathbb{S}^{N-1}, I=$ $\partial D$. We also set $\Sigma=A$. For simplicity, throughout this subsection, we also set the dimensional parameter $R_{0}$ equal to 1 . We set $Q=B_{1} \times(\pi / 2,3 \pi / 2) \subset \mathbb{R}^{N+1}$ and $\Gamma=A \times(\pi / 2,3 \pi / 2)$.

We wish to remark that we could equivalently consider the time interval $(0, \pi)$ or $(0,1)$ instead. We have chosen $(\pi / 2,3 \pi / 2)$ only because it will turn out to be convenient for the purpose of the proof.

The following Hilbert spaces will be used. The space $H=H_{.0}^{3 / 2,3 / 4}(\Gamma)$, its dual $H^{\prime}=H_{1}=H^{-3 / 2,-3 / 4}(\Gamma)$, and $H_{0}=H^{1 / 2,1 / 4}(\Gamma)$. We consider now the interpolation spaces between $H_{0}$ and $H_{1}$. For what concerns interpolation we refer again to [16]. For any $\theta, 0 \leq \theta \leq 1$, we define $H_{\theta}$ as $\left[H_{0}, H_{1}\right]_{\theta}$, where this denotes the interpolation at level $\theta$ between the two spaces $H_{0}$ and $H_{1}$. The norm in $H_{\theta}$ will be denoted by $\|\cdot\|_{\theta}$. First, we notice that for any $\theta, 0 \leq \theta \leq 1$, there exists a constant $C_{\theta}$, which depends on $\theta$ only, such that the following interpolation inequality holds for any $\psi \in H_{0}$

$$
\|\psi\|_{\theta} \leq C_{\theta}\|\psi\|_{0}^{1-\theta}\|\psi\|_{1}^{\theta} .
$$


By using the interpolation properties of fractional order Sobolev spaces on $\Gamma$, see [16], we can characterize $H_{\theta}$ as follows

$$
H_{\theta}= \begin{cases}H^{2(1 / 4-\theta), 1 / 4-\theta}(\Gamma) & \text { if } 0 \leq \theta \leq 1 / 4 \\ H^{-2(\theta-1 / 4),-(\theta-1 / 4)}(\Gamma) & \text { if } 1 / 4 \leq \theta \leq 1, \theta \neq 3 / 4 .\end{cases}
$$

Let us notice the interesting case of $\theta=1 / 4$, where we have $H_{\theta}=L^{2}(\Gamma)$, and that $H_{3 / 4}$ does not coincide with $H^{-1,-1 / 2}(\Gamma)$.

Let us fix an integer $m \geq 2$ and positive constants $\delta, b$ and $r$.

To any strictly positive function $g$ defined on $r \mathbb{S}^{N-1}=\partial B_{r}$, we denote its radial subgraph as $\operatorname{subgraph}_{\text {rad }}(g)=\left\{y \in \mathbb{R}^{N}: y=\rho \omega, 0 \leq \rho \leq\right.$ $\left.g(r \omega), \omega \in \mathbb{S}^{N-1}\right\}$. Then, $X_{m b \delta}\left(\bar{B}_{r}\right)$ denotes the set given by $\left\{\operatorname{subgraph}_{\text {rad }}(g)\right.$ : $g \in C^{\infty}\left(r \mathbb{S}^{N-1}\right),\|g\|_{C^{m}\left(r \mathbb{S}^{N-1}\right)} \leq b$ and $\left.r \leq g \leq r+\delta\right\}$.

Let us consider the metric space $(X, d)=\left(X_{m b \frac{1}{4}}\left(\bar{B}_{1 / 2}\right), d_{\mathcal{H}}\right)$ where $d_{\mathcal{H}}$ denotes the Hausdorff distance. Let us notice that every $D \in X$ is closed, is star-shaped with respect to the origin and satisfies $\bar{B}_{1 / 2} \subset D \subset \bar{B}_{3 / 4}$.

For any $D \in X$, we set $Q(D)=\left(B_{1} \backslash D\right) \times(\pi / 2,3 \pi / 2), \Gamma(D)=\partial D \times$ $(\pi / 2,3 \pi / 2)$. If $D=\emptyset$, then we set $Q(D)=Q$ and $\Gamma(D)=\emptyset$.

For any $D \in X \cup\{\emptyset\}$, we consider the operator $\mathcal{D}(D): H \mapsto H_{0}$ which is defined as follows. For any $\psi \in H$, let $u \in H^{2,1}(Q(D))$ be the solution to

$$
\begin{cases}\partial_{t} u-\Delta u=0 & \text { in } Q(D), \\ u(x, \pi / 2)=0 & x \in \Omega \backslash D, \\ u=0 & \text { on } \Gamma(D), \\ u=\psi & \text { on } \Gamma .\end{cases}
$$

Then, for any $\psi \in H$, we set

$$
\mathcal{D}(D) \psi=\left.\frac{\partial u}{\partial \nu}\right|_{\Gamma}, \quad u \text { solution to }(2.14) .
$$

We have that Theorems 4.3 and 6.2 in [16, Chapter 4] imply, respectively, existence and uniqueness of a solution $u \in H^{2,1}(Q(D))$ to (2.14) and its continuous dependence from the boundary datum $\psi \in H$. Finally, by the trace theorem [16, Chapter 4, Theorem 2.1], we can conclude that, for any $D \in X \cup\{\emptyset\}$, the operator $\mathcal{D}(D): H \mapsto H_{0}$ is linear and bounded. We can also consider $\mathcal{D}(D)$ as a linear and bounded operator between $H$ and $H^{\prime}=H_{1}$, by setting

$$
\langle\mathcal{D}(D) \psi, \phi\rangle_{H^{\prime}, H}=\left\langle\left.\frac{\partial u}{\partial \nu}\right|_{\Gamma}, \phi\right\rangle_{H^{\prime}, H}=\int_{\Gamma} \frac{\partial u}{\partial \nu} \phi, \quad \text { for any } \psi, \phi \in H,
$$

where $u$ solves $(2.14)$ and $\langle\cdot, \cdot\rangle_{H^{\prime}, H}$ is the duality pairing between $H^{\prime}$ and $H$.

Let us remark that the operator $\mathcal{D}$ is usually referred to as the Dirichlet-toNeumann map. We are in the position of stating the instability result.

Theorem 2.5 Let us fix an integer $m \geq 2$ and a positive constant $b$. Let $(X, d)=\left(X_{m b \frac{1}{4}}\left(\bar{B}_{1 / 2}\right), d_{\mathcal{H}}\right)$. Then there exists a positive constant $\delta_{1}$, depending on $m$ and $b$ only, such that for every $\delta, 0<\delta<\delta_{1}$, we can find $D_{1}, D_{2} \in X$ satisfying

$$
d\left(D_{i}, \bar{B}_{1 / 2}\right) \leq \delta, \text { for any } i=1,2 ; \quad d\left(D_{1}, D_{2}\right) \geq \delta ;
$$

and, for any $\theta, 0 \leq \theta \leq 1$,

$$
\left\|\mathcal{D}\left(D_{1}\right)-\mathcal{D}\left(D_{2}\right)\right\|_{\mathcal{L}\left(H, H_{\theta}\right)} \leq K \exp \left(-\theta \delta^{-\frac{N-1}{2 m(2 N+1)}}\right),
$$

where $K$ is a constant depending on $m, b$ and $\theta$ only. 


\section{Quantitative estimates of unique continuation}

We shall prove some Carleman estimates and we shall apply them to solutions to parabolic equations and we shall obtain an optimal three cylinder inequality at the boundary, a stability estimate for Cauchy problems and an estimate of smallness propagation. Since the Carleman estimates are obtained by the technique employed in [9] we shall adopt the notation used in that paper.

Let $\left\{g^{i j}(x, t)\right\}$ be a symmetric $N \times N$ matrix whose entries are real functions. When $\xi \in \mathbb{R}^{N}$ and $(x, t),(y, s) \in \mathbb{R}^{N+1}$ assume that

$$
\lambda^{-1}|\xi|^{2} \leq \sum_{i, j=1}^{N} g^{i j}(x, t) \xi_{i} \xi_{j} \leq \lambda|\xi|^{2}
$$

and

$$
\left(\sum_{i, j=1}^{N}\left(g^{i j}(x, t)-g^{i j}(y, s)\right)^{2}\right)^{1 / 2} \leq \Lambda(|x-y|+|t-s|) .
$$

Let $q_{0}$ be a given positive number and let $L$ be the following parabolic operator

$$
L u=\partial_{i}\left(g^{i j}(x, t) \partial_{j} u\right)-q_{0} \partial_{t} u .
$$

For any positive numbers $r$ and $t_{0}$ we set $Q_{r}^{t_{0}}=B_{r} \times\left(-t_{0}, t_{0}\right), \tilde{Q}_{r}^{t_{0}}=$ $\left(B_{r} \backslash\{0\}\right) \times\left(-t_{0}, t_{0}\right)$. When $h$ is a $C^{0,1}\left(\mathbb{R}^{N-1}\right)$ function such that $h(0)=0$, we shall denote by $\Gamma_{h}$ the set

$$
\Gamma_{h}=\left\{(x, t) \in \mathbb{R}^{N+1}: x_{N}=h\left(x^{\prime}\right)\right\},
$$

we shall denote by $\Gamma_{h, r}^{t_{0}}$ the set $\Gamma_{h} \cap Q_{r}^{t_{0}}$ and we shall denote by $Q_{h, r}^{t_{0}}$ the set

$$
Q_{h, r}^{t_{0}}=\left\{(x, t) \in Q_{r}^{t_{0}}: x_{N}>h\left(x^{\prime}\right)\right\} .
$$

If $x=\left(x^{\prime}, h\left(x^{\prime}\right)\right)$ we denote by $\nu(x)$, or simply by $\nu$, the unit vector of $\mathbb{R}^{N}$

$$
\nu(x)=\frac{\left(-\nabla_{x^{\prime}} h\left(x^{\prime}\right), 1\right)}{\sqrt{1+\left|\nabla_{x^{\prime}} h\left(x^{\prime}\right)\right|^{2}}} .
$$

To simplify the notation we shall use some of the standard notation in Riemannian geometry, but we shall always drop the corresponding volume element in the definition of the Laplace-Beltrami operator associated to a Riemannian metric. We do this because it simplifies the formulas appearing in the proofs of the following lemmas, especially when the metric is allowed to depend on the time variable and we make use of partial integration with respect to this variable.

In particular, if $g(x, t)=\left\{g_{i j}(x, t)\right\}_{i, j=1}^{N}$ denotes the inverse matrix of the matrix of coefficients of $L$, we have $g^{-1}(x, t)=\left\{g^{i j}(x, t)\right\}_{i, j=1}^{N}$, and, for any function $f$ and any two variable vector fields $\xi$ and $\eta$, we set

$$
\begin{aligned}
& \xi \cdot \eta=\sum_{i, j=1}^{N} g_{i j}(x, t) \xi_{i} \eta_{j}, \quad|\xi|^{2}=\xi \cdot \xi \\
& \nabla f=g^{-1} \nabla_{x} f, \quad \operatorname{div}(\xi)=\sum_{i=1}^{N} \partial_{i} \xi^{i}, \quad \Delta f=\operatorname{div}(\nabla f) .
\end{aligned}
$$


When $h$ is a $C^{0,1}\left(\mathbb{R}^{N-1}\right)$ function and $x=\left(x^{\prime}, h\left(x^{\prime}\right)\right)$ we denote by $\mathbf{n}(x, t)$, or simply by $\mathbf{n}$, the vector

$$
\mathbf{n}(x, t)=g^{-1}(x, t) \nu(x) .
$$

With this notation we have

$$
L u=\Delta u-q_{0} \partial_{t} u .
$$

For the sake of brevity, in the sequel we shall denote, respectively, by $\int$ the integral over $Q_{1}^{1}$ with respect to the Lebesgue measure $\mathrm{d} X=\mathrm{d} x \mathrm{~d} t$ and by $\int_{\Gamma_{h, 1}^{1}}$ the integral over $\Gamma_{h, 1}^{1}$ with respect to the $N$-dimensional surface measure. In Theorem 3.1 below we shall adopt the following notation. We denote

$$
\rho(x, t)=\left(\sum_{i, j=1}^{N} g_{i j}(0, t) x_{i} x_{j}\right)^{1 / 2} .
$$

For positive numbers $a$ and $\mu$ to be chosen later we set

$$
\sigma(x, t)=\rho(x, t)-a(\rho(x, t))^{\beta}\left(\sum_{j=1}^{N} g_{N j}(0, t) x_{j}\right)
$$

and

$$
w(x, t)=\varphi(\sigma(x, t)), \quad \text { where } \varphi(s)=s \exp \left(\int_{0}^{s} \frac{\mathrm{e}^{-\mu \tau^{\beta}}-1}{\tau} \mathrm{d} \tau\right) .
$$

Observe that $\rho$ is the distance function from $x=0$ associated to the metric $\sum_{i, j=1}^{N} g_{i j}(0, t) \mathrm{d} x_{i} \mathrm{~d} x_{j}$ and $\sigma$ is a perturbation of $\rho$ satisfying

$$
|\sigma(x, t)-\rho(x, t)| \leq C a(\rho(x, t))^{1+\beta},
$$

where $C$ depends on $\lambda$ only.

Theorem 3.1 Assume that the parabolic operator $L$ satifies the conditions (3.2) and (3.3) and let us take $h \in C^{1, \beta}\left(B_{1}^{\prime}\right)$ such that $h(0)=\left|\nabla_{x^{\prime}} h(0)\right|=0$ and $\|h\|_{C^{1, \beta}\left(B_{1}^{\prime}\right)} \leq E$, with $0<\beta \leq 1$ and $E>0$. Then, there are constants $0<d<$ $1, \mu_{0}>0, a_{0}>0$ and $C>1$ depending on $\lambda, \Lambda, E$ and $\beta$ only such that, for any $\alpha \geq C\left(q_{0}+1\right)$ and any $u \in C^{1}\left(\bar{Q}_{h, 1}^{1}\right) \cap C^{\infty}\left(Q_{h, 1}^{1}\right)$ such that $u=0$ on $\Gamma_{h, 1}^{1}$ and $\operatorname{supp} u \subset Q_{d}^{1}$, the following inequality holds

$$
C \int w^{2-2 \alpha}(L u)^{2} \geq \int\left(\alpha w^{\beta-2 \alpha}|\nabla u|^{2}+\alpha^{3} w^{\beta-2-2 \alpha} u^{2}\right),
$$

where $w$ is defined by (3.5) and (3.6) with $a=a_{0}$ and $\mu=\mu_{0}$.

We begin the proof of the theorem above by setting, for any function $w \in$ $C^{2,1}\left(Q_{1}^{1}\right)$ such that $w,|\nabla w|>0$ in $Q_{h, 1}^{1}$, the operator $L_{\alpha}(f)=w^{-\alpha} L\left(w^{\alpha} f\right)$. We have

$$
L_{\alpha}(f)=\Delta f+\alpha^{2} \frac{|\nabla w|^{2}}{w^{2}} f-\alpha q_{0}\left(\partial_{t} \log w\right) f+2 \alpha \frac{|\nabla w|^{2}}{w^{2}} A(f)-q_{0} \partial_{t} f,
$$


where

$$
A(f)=w \frac{\nabla w \cdot \nabla f}{|\nabla w|^{2}}+\frac{1}{2} F_{w}^{g} f, \quad F_{w}^{g}=\frac{w \Delta w-|\nabla w|^{2}}{|\nabla w|^{2}} .
$$

We shall denote by $\mathcal{M}_{w}^{g}$ the symmetric $N \times N$ matrix

$$
\mathcal{M}_{w}^{g}=\frac{1}{2}\left\{M_{i j}+M_{j i}\right\}_{i, j=1}^{N},
$$

where, using the summation notation of repeated indices,

$$
M_{i j}=\frac{1}{2} \operatorname{div}\left(\frac{w \nabla w}{|\nabla w|^{2}}\right) \delta_{i j}-\partial_{j}\left(\frac{w g^{i k} \partial_{k} w}{|\nabla w|^{2}}\right)+\frac{1}{2} g_{j h} \frac{w g^{k l} \partial_{l} w}{|\nabla w|^{2}} \partial_{k} g^{h i}-\frac{1}{2} F_{w}^{g} \delta_{i j} .
$$

The following lemmas hold true, [9].

Lemma 3.2 Let $\rho$ be defined by (3.4), let $\varphi$ be a positive nondecreasing function on $(0,+\infty)$. Let $w$ be a function of $C^{2,1}\left(Q_{1}^{1}\right)$ class such that $w,|\nabla w|>0$ in $Q_{h, 1}^{1}$. Let

$$
\phi(s)=\varphi(s) /\left(s \varphi^{\prime}(s)\right) .
$$

Then the symmetric matrix $\mathcal{M}_{w}^{g}$ satisfies $\mathcal{M}_{w}^{g} \nabla w=0$ and the following facts hold

$$
\begin{aligned}
& F_{\varphi(w)}^{g}=\phi(w) F_{w}^{g}-w \phi^{\prime}(w), \quad \mathcal{M}_{\varphi(w)}^{g}=\phi(w) \mathcal{M}_{w}^{g}+w \phi^{\prime}(w)\left(\mathcal{I}-\frac{\nabla w \otimes \nabla w}{|\nabla w|^{2}} g\right), \\
& F_{\rho}^{g(0, t)}=N-2, \quad \mathcal{M}_{\rho}^{g(0, t)}=0 .
\end{aligned}
$$

Lemma 3.3 Let $w$ be a function of $C^{2,1}\left(Q_{1}^{1}\right)$ class such that $w,|\nabla w|>0$ in $Q_{h, 1}^{1}$. Then, for any $\alpha \geq 1$ and any $u \in C^{1}\left(\bar{Q}_{1}^{1}\right) \cap C^{\infty}\left(Q_{1}^{1}\right)$ such that $u=0$ on $\Gamma_{h, 1}^{1}$ and $\operatorname{supp} u \subset Q_{1}^{1}$, the following inequality holds

$$
\begin{gathered}
\int \frac{w^{2}}{|\nabla w|^{2}}\left(L_{\alpha} f\right)^{2}-\mathcal{B}_{w}(f) \geq 4 \alpha \int \mathcal{M}_{w}^{g} \nabla f \cdot \nabla f+\alpha \int F_{w}^{g} \Delta\left(f^{2}\right)-2 q_{0} \int F_{w}^{g} f \partial_{t} f \\
+2 \alpha \int \frac{|\nabla w|^{2}}{w^{2}} A(f)^{2}+\frac{q_{0}^{2}}{\alpha} \int \frac{w^{2}}{|\nabla w|^{2}}\left(\partial_{t} f\right)^{2}-q_{0} \int|\nabla f|^{2} \partial_{t} \frac{w^{2}}{|\nabla w|^{2}} \\
-q_{0} \int \frac{w^{2}}{|\nabla w|^{2}} \partial_{t} g^{i j} \partial_{i} f \partial_{j} f-2 q_{0} \int \frac{w^{2} \nabla|\nabla w|^{2} \cdot \nabla f}{|\nabla w|^{4}} \partial_{t} f \\
-4 \alpha^{2} q_{0} \int\left(\partial_{t} \log w\right) A(f) f+2 \alpha q_{0}^{2} \int\left(\partial_{t} \log w\right) \frac{w^{2}}{|\nabla w|^{2}} f \partial_{t} f
\end{gathered}
$$

where

$$
\mathcal{B}_{w}(f)=2 \alpha \int_{\Gamma_{h, 1}^{1}} \frac{w \nabla w \cdot \mathbf{n}}{|\nabla w|^{2}|\mathbf{n}|^{2}}(\nabla f \cdot \mathbf{n})^{2} .
$$

Proof of Theorem 3.1. Let $w$ be defined by (3.6). First of all we choose $a$ in such a way that $\nabla w \cdot \mathbf{n} \geq 0$ in a neighbourhood of 0 . Denoting by $(\cdot \cdot \cdot)$ the Euclidean scalar product we have

$$
\nabla w \cdot \mathbf{n}=\varphi^{\prime}(\sigma)\left(\left(\left(g^{-1}(x, t)-g^{-1}(0, t)\right) \nabla \sigma \mid \nu\right)+\left(g^{-1}(0, t) \nabla \sigma \mid \nu\right)\right)
$$


and

$$
\begin{aligned}
\left(g^{-1}(0, t) \nabla \sigma \mid \nu\right) & =a \frac{(\rho(x, t))^{\beta}}{\sqrt{1+\left|\nabla_{x^{\prime}} h\right|^{2}}}+\frac{\left(x^{\prime} \nabla_{x^{\prime}} h-h\left(x^{\prime}\right)\right)}{\rho(x, t) \sqrt{1+\left|\nabla_{x^{\prime}} h\right|^{2}}} \\
& -\frac{a \beta\left(x^{\prime} \nabla_{x^{\prime}} h-h\left(x^{\prime}\right)\right)}{\sqrt{1+\left|\nabla_{x^{\prime}} h\right|^{2}}}\left(\sum_{j=1}^{N} g_{N j}(0, t) x_{j}\right)(\rho(x, t))^{\beta-2} .
\end{aligned}
$$

Let us now choose $a=2 E \lambda^{-(\beta+1) / 2}$. By (3.16) we obtain

$$
\left(g^{-1}(0, t) \nabla \sigma \mid \nu\right) \geq C^{-1}\left|x^{\prime}\right|^{\beta}, \quad \text { for every } x^{\prime} \in B_{1 / C}^{\prime},
$$

where $C>1$ depends on $\lambda, E$ and $\beta$ only. With this value of $a$ we also have

$$
\left|\left(\left(g^{-1}(x, t)-g^{-1}(0, t)\right) \nabla \sigma \mid \nu\right)\right| \leq C|x|, \quad \text { on } \Gamma_{h, 1}^{1},
$$

where $C$ depends on $\lambda, \Lambda, E$ and $\beta$ only.

By (3.15), (3.14) and the inequalities above, we obtain

$$
\mathcal{B}_{w}(f) \geq 0,
$$

for all $f \in C^{1}\left(\bar{Q}_{h, 1}^{1}\right) \cap C^{\infty}\left(Q_{h, 1}^{1}\right)$ such that $f=0$ on $\Gamma_{h, 1}^{1}$ and $\operatorname{supp} f \subset Q_{1 / C}^{1}$, where $C>1$ depends on $\lambda, \Lambda, E$ and $\beta$ only.

With the previous choice of $a$ and for a fixed number $\mu \geq 1$, we have $\phi(s)=$ $\mathrm{e}^{\mu s^{\beta}}$, where $\phi$ is defined in Lemma 3.2. Moreover the following properties can be easily checked on $Q_{1 / C}^{1}$ for some constant $C>1$ depending on $\lambda, \Lambda, E, \beta$ and $\mu$ only

$$
\begin{array}{lll}
|x| / C \leq \sigma \leq C|x|, & \sigma / C \leq w \leq C \sigma, & 1 / C \leq|\nabla w| \leq C, \\
\left|\partial_{t}\left(w^{2} /|\nabla w|^{2}\right)\right| \leq C w^{2}, & \left|\partial_{t} \phi\right| \leq C w^{\beta}, & \left.|\nabla| \nabla w\right|^{2} \mid \leq C w^{\beta-1}, \\
|\Delta \phi| \leq C w^{\beta-2}, & \left|F_{w}^{g}\right| \leq C, & \left|\partial_{t} \log w\right| \leq C .
\end{array}
$$

In order to estimate from below the first integral on the right hand side of (3.13), we observe that if we denote

$$
\tilde{\nabla} f=\nabla f-\frac{(\nabla \sigma \cdot \nabla f)}{|\nabla \sigma|^{2}} \nabla \sigma=\nabla f-\frac{(\nabla w \cdot \nabla f)}{|\nabla w|^{2}} \nabla w,
$$

then from Lemma 3.2 we have

$$
\mathcal{M}_{w}^{g} \nabla f \cdot \nabla f=\sigma \phi^{\prime}|\tilde{\nabla} f|^{2}+\phi \mathcal{M}_{\sigma}^{g} \tilde{\nabla} f \cdot \tilde{\nabla} f .
$$

Now, from Lemma $3.2, \mathcal{M}_{\rho}^{g(0, t)}=0$, and by straightforward calculations we have

$$
\left|\mathcal{M}_{\sigma}^{g}\right|=\left|\mathcal{M}_{\sigma}^{g}-\mathcal{M}_{\sigma}^{g(0, t)}\right|+\left|\mathcal{M}_{\sigma}^{g(0, t)}-\mathcal{M}_{\rho}^{g(0, t)}\right| \leq C_{0} \sigma^{\beta}, \quad \text { for any } \sigma \leq 1 / C_{0},
$$

where $C_{0}>1$ depends on $\lambda, \Lambda, E$ and $\beta$ only (note that $C_{0}$ does not depend on $\mu$ ).

By (3.19) and (3.20) we have, when $\operatorname{supp} f \subset Q_{1 / C_{0}}^{1}$,

$$
\int \mathcal{M}_{w}^{g} \nabla f \cdot \nabla f \geq \int\left(\sigma \phi^{\prime}-C_{0} \sigma^{\beta} \phi\right)|\tilde{\nabla} f|^{2} .
$$


Now we estimate from below the second and third integrals on the right hand side of (3.13). From Lemma 3.2 we have

$$
F_{w}^{g}=(N-2) \phi+\left(B \phi-\sigma \phi^{\prime}\right),
$$

where $B=F_{\sigma}^{g}-(N-2)$.

From Lemma $3.2, F_{\rho}^{g(0, t)}=N-2$, hence

(3.23) $|B(x, t)| \leq\left|F_{\sigma}^{g}-F_{\sigma}^{g(0, t)}\right|+\left|F_{\sigma}^{g(0, t)}-F_{\rho}^{g(0, t)}\right| \leq C_{0} \sigma^{\beta}$, for any $\sigma \leq 1 / C_{0}$,

where $C_{0}>1$ depends on $\lambda, \Lambda, E$ and $\beta$ only.

The identity

$$
|\nabla f|^{2}=|\tilde{\nabla} f|^{2}+\frac{(\nabla w \cdot \nabla f)^{2}}{|\nabla w|^{2}}
$$

(3.22) and the divergence theorem imply that

$$
\begin{aligned}
\int F_{w}^{g} \Delta\left(f^{2}\right)= & (N-2) \int f^{2} \Delta(\phi)+2 \int\left(B \phi-\sigma \phi^{\prime}\right) f \Delta f \\
& +2 \int\left(B \phi-\sigma \phi^{\prime}\right)|\tilde{\nabla} f|^{2}+2 \int\left(B \phi-\sigma \phi^{\prime}\right) \frac{(\nabla w \cdot \nabla f)^{2}}{|\nabla w|^{2}}
\end{aligned}
$$

and

$$
\int F_{w}^{g} f \partial_{t} f=-\frac{(N-2)}{2} \int f^{2} \partial_{t} \phi+\int\left(B \phi-\sigma \phi^{\prime}\right) f \partial_{t} f .
$$

By the identity (3.9), we obtain the following formula for the second integral on the right hand side of $(3.25)$

$$
\begin{aligned}
& 2 \int\left(B \phi-\sigma \phi^{\prime}\right) f \Delta f= \\
& 2 \int\left(B \phi-\sigma \phi^{\prime}\right) w^{-\alpha} f L_{\alpha} f+2 \alpha^{2} \int\left(\sigma \phi^{\prime}-B \phi\right) \frac{|\nabla w|^{2}}{w^{2}} f^{2} \\
& \quad+2 \int\left(\sigma \phi^{\prime}-B \phi\right) f\left(2 \alpha \frac{|\nabla w|^{2}}{w^{2}} A(f)-q_{0} \partial_{t} f-q_{0} \alpha\left(\partial_{t} \log w\right) f\right) .
\end{aligned}
$$

By (3.21), (3.25), (3.26) and (3.27) we obtain, for any $\alpha \geq 1$,

$$
\begin{aligned}
& 4 \alpha \int \mathcal{M}_{w}^{g} \nabla f \cdot \nabla f+\alpha \int F_{w}^{g} \Delta\left(f^{2}\right)-2 q_{0} \int F_{w}^{g} f \partial_{t} f \geq \\
& 2 \alpha \int\left(\sigma \phi^{\prime}-2 C_{0} \sigma^{\beta} \phi+B \phi\right)|\tilde{\nabla} f|^{2}+2 \alpha^{3} \int\left(\sigma \phi^{\prime}-B \phi\right) \frac{|\nabla w|^{2}}{w^{2}} f^{2}-\mathcal{R}_{1},
\end{aligned}
$$

where

$$
\begin{aligned}
& \mathcal{R}_{1}=2 \alpha \int\left(B \phi-\sigma \phi^{\prime}\right) w^{-\alpha} f L_{\alpha} f+(N-2) \alpha \int f^{2} \Delta(\phi) \\
&+2 \alpha \int\left(\sigma \phi^{\prime}-B \phi\right) f(\left(2 \alpha \frac{|\nabla w|^{2}}{w^{2}} A(f)-q_{0} \partial_{t} f-q_{0} \alpha\left(\partial_{t} \log w\right) f\right) \\
&+(N-2) q_{0} \int f^{2} \partial_{t} \phi-2 q_{0} \int\left(B \phi-\sigma \phi^{\prime}\right) f \partial_{t} f
\end{aligned}
$$


By (3.18) and Young inequality we obtain

$$
\begin{aligned}
\mathcal{R}_{1} & \leq \frac{1}{2} \int \frac{w^{2}}{|\nabla w|^{2}}\left(L_{\alpha} f\right)^{2}+C \int\left(\alpha^{3} w^{-2+3 \beta / 2}+\alpha w^{-2+\beta}+\alpha^{2}\left(w^{-2+2 \beta}+q_{0}\right)\right) f^{2} \\
& +C \alpha \int w^{-2+\beta / 2}(A(f))^{2}+C \alpha \int w^{\beta} \frac{(\nabla w \cdot \nabla f)^{2}}{|\nabla w|^{2}}+\frac{q_{0}^{2}}{2 \alpha} \int \frac{w^{2}}{|\nabla w|^{2}}\left(\partial_{t} f\right)^{2},
\end{aligned}
$$

where $C$ depends on $\lambda, \Lambda, E, \beta$ and $\mu$ only.

Now, let us choose $\mu=4 C_{0}$. With this choice of $\mu$ and by (3.18) we obtain the following estimate from below for the first term on the right hand side of (3.28)

$$
2 \alpha \int\left(\sigma \phi^{\prime}-2 C_{0} \sigma^{\beta} \phi+B \phi\right)|\tilde{\nabla} f|^{2} \geq C_{0} \alpha \int w^{\beta}|\tilde{\nabla} f|^{2}, \quad \text { for any } \alpha \geq 1,
$$

where $C_{0}$ depends on $\lambda, \Lambda, E$ and $\beta$ only.

On the other hand, by (3.10) we obtain

$$
\begin{aligned}
\int w^{\beta} \frac{(\nabla w \cdot \nabla f)^{2}}{|\nabla w|^{2}}=\int \frac{|\nabla w|^{2}}{w^{2-\beta}} & \left(A(f)-F_{w}^{g} f\right)^{2} \\
& \leq 2 \int \frac{|\nabla w|^{2}}{w^{2-\beta}}(A(f))^{2}+\frac{1}{2} \int \frac{|\nabla w|^{2}}{w^{2-\beta}}\left(F_{w}^{g}\right)^{2} f^{2}
\end{aligned}
$$

This inequality and the second formula in (3.24) yield to

$$
\int w^{\beta}|\tilde{\nabla} f|^{2} \geq C^{-1} \int w^{\beta}|\nabla f|^{2}-C \int \frac{|\nabla w|^{2}}{w^{2-\beta}}(A(f))^{2}-C \int \frac{|\nabla w|^{2}}{w^{2-\beta}}\left(F_{w}^{g}\right)^{2} f^{2},
$$

where $C>1$ depends on $\lambda, \Lambda, E$ and $\beta$ only.

The inequality above, (3.13), (3.17), (3.28) and (3.30) yield to

$$
\begin{array}{r}
\int \frac{w^{2}}{|\nabla w|^{2}}\left(L_{\alpha} f\right)^{2} \geq C^{-1} \alpha \int w^{\beta}|\nabla f|^{2}+\alpha^{3} \int\left(C^{-1}-C / \alpha^{2}\right) \frac{|\nabla w|^{2}}{w^{2-\beta}} f^{2} \\
+\alpha \int\left(2-C w^{\beta}\right) \frac{|\nabla w|^{2}}{w^{2}}(A(f))^{2}+\frac{q_{0}^{2}}{\alpha} \int \frac{w^{2}}{|\nabla w|^{2}}\left(\partial_{t} f\right)^{2}-\mathcal{R}_{2}
\end{array}
$$

where

$$
\begin{aligned}
\mathcal{R}_{2}=\mathcal{R}_{1}-q_{0} \int \frac{w^{2}}{|\nabla w|^{2}} \partial_{t} g^{i j} \partial_{i} f \partial_{j} f-2 q_{0} \int \frac{w^{2} \nabla|\nabla w|^{2} \cdot \nabla f}{|\nabla w|^{4}} \partial_{t} f \\
\quad-4 \alpha^{2} q_{0} \int\left(\partial_{t} \log w\right) A(f) f+2 \alpha q_{0}^{2} \int\left(\partial_{t} \log w\right) \frac{w^{2}}{|\nabla w|^{2}} f \partial_{t} f .
\end{aligned}
$$

Using (3.29) and Young inequality to estimate $\mathcal{R}_{2}$ from above, by (3.18) and (3.31) we obtain, for any $\alpha \geq C$ and $\operatorname{supp} f \subset Q_{1 / C}^{1}$,

$$
\begin{aligned}
\frac{1}{2} \int \frac{w^{2}}{|\nabla w|^{2}}\left(L_{\alpha} f\right)^{2} \geq C^{-1} \alpha \int w^{\beta}|\nabla f|^{2}+C^{-1} \alpha^{3} \int w^{-2+\beta} f^{2} \\
+C^{-1} \alpha \int w^{2}(A(f))^{2}+\frac{q_{0}^{2}}{2 \alpha} \int \frac{w^{2}}{|\nabla w|^{2}}\left(\partial_{t} f\right)^{2}
\end{aligned}
$$


where $C>1$ depends on $\lambda, \Lambda, E$ and $\beta$ only.

Finally, recalling that $f=w^{-\alpha} u$ and (3.9), we easily obtain (3.8).

By the Carleman estimates, we can obtain the following three cylinder inequalities. In Theorem 3.4 we state the three cylinder inequality in the interior, proved in [9], in Theorem 3.5 we state the three cylinder inequality at the boundary. We shall omit its proof because it is analogous to the one of Theorem 15 in [21].

Theorem 3.4 (Three Cylinder Inequality in the Interior) Let $T_{1}$ and $R$ be positive numbers such that $T_{1} \in(0, T], R \in\left(0, R_{0}\right]$. Let $\kappa$ be a symmetric $N \times N$ real matrix satisfying (2.7) and (2.8). Let $u \in H^{2,1}\left(D_{R}^{T_{1}}\right)$ satisfy

$$
\partial_{t} u-\operatorname{div}(\kappa(x, t) \nabla u)=0 \text { in } D_{R}^{T_{1}}, \quad u(x, 0)=0 .
$$

Then, there exist constants $s_{1} \in(0,1)$ and $C>1$ depending on $\lambda$ and $\Lambda$ only such that, for any $\rho_{1}, \rho_{2}$ and $\tau$ satisfying $0<\rho_{1}<\rho_{2}<s_{1}^{2} R, \tau \in\left(0, T_{1}\right)$, the following inequality holds

$$
\|u\|_{L^{2}\left(D_{\rho_{2}}^{T_{1}-\tau}\right)} \leq \gamma\left(\frac{C R}{\rho_{2}}\right)^{\gamma}\|u\|_{L^{2}\left(D_{\rho_{1}}^{T_{1}}\right)}^{\vartheta}\|u\|_{L^{2}\left(D_{R}^{T_{1}}\right)}^{1-\vartheta},
$$

where

$$
\vartheta=\frac{\log \frac{s_{1} R}{\rho_{2}}}{C \log \frac{C R}{\rho_{1}}} \quad \text { and } \quad \gamma=C\left(\frac{R^{2}}{T_{1}}+\frac{T_{1}}{\tau}\right)^{C}
$$

Theorem 3.5 (Three Cylinder Inequality at the Boundary) $L e t T_{1}$ and $R$ be positive numbers such that $T_{1} \in(0, T], R \in\left(0, R_{0}\right]$. Let $\kappa$ be a symmetric $N \times N$ real matrix satisfying (2.7) and (2.8) and $\psi$ be a function of $C^{1, \beta}$ class with constants $E, R_{0}$ such that $\psi(0)=0$. Let $u \in H^{1,1}\left(D_{\psi, R}^{T_{1}}\right) \cap H_{l o c}^{2,1}\left(D_{\psi, R}^{T_{1}}\right)$ be a solution to

$$
\partial_{t} u-\operatorname{div}(\kappa(x, t) \nabla u)=0 \text { in } D_{\psi, R}^{T_{1}}, \quad u=0 \text { on } \Gamma_{\psi} \cap D_{R}^{T_{1}}, \quad u(x, 0)=0 .
$$

Then, there exist constants $s_{1} \in(0,1)$ and $C>1$ depending on $\lambda, \Lambda, E$ and $\beta$ only such that, for any $\rho_{1}, \rho_{2}$ and $\tau$ satisfying $0<\rho_{1}<\rho_{2}<s_{1}^{2} R, \tau \in\left(0, T_{1}\right)$, the following inequality holds

$$
\|u\|_{L^{2}\left(D_{\psi, \rho_{2}}^{T_{1}-\tau}\right)} \leq \gamma\left(\frac{C R}{\rho_{2}}\right)^{\gamma}\|u\|_{L^{2}\left(D_{\psi, \rho_{1}}^{T_{1}}\right)}^{\vartheta}\|u\|_{L^{2}\left(D_{\psi, R}^{T_{1}}\right)}^{1-\vartheta},
$$

where $\vartheta$ and $\gamma$ are as in (3.33).

We now turn our attention to the stability estimates for Cauchy problems and to smallness propagation estimates.

Theorem 3.6 (Stability Estimate for Cauchy Problem) Let $\delta, T_{1}$ and $R$ be positive numbers such that $T_{1} \in(0, T], R \in\left(0, R_{0}\right]$. Let $\kappa$ be a symmetric $N \times N$ real matrix satisfying (2.7) and (2.8) and $\psi$ be a function of $C^{0,1}$ class 
with constants $E, R_{0}$ such that $\psi(0)=0$. Let $u \in H^{1,1}\left(D_{\psi, R}^{T_{1}}\right) \cap H_{l o c}^{2,1}\left(D_{\psi, R}^{T_{1}}\right)$ be a solution to

$$
\partial_{t} u-\operatorname{div}(\kappa(x, t) \nabla u)=0 \text { in } D_{\psi, R}^{T_{1}}, \quad u=0 \text { on } \Gamma_{\psi} \cap D_{R}^{T_{1}}, \quad u(x, 0)=0
$$

and satisfy

$$
\frac{1}{R_{0}^{N-1} T} \int_{\Gamma_{\psi} \cap D_{R}^{T_{1}}}(\kappa \nabla u \mid \nu)^{2} \leq \delta^{2} .
$$

Then, there exists a constant $C>1$ depending on $\lambda, \Lambda$ and $E$ only such that, for any $\rho_{1}, \rho_{2}$ and $\tau$ satisfying $0<\rho_{2} \leq C^{-1} R, 0<\rho_{1}<C^{-1} \rho_{2}, \tau \in\left(0, T_{1}\right)$, the following inequality holds

$$
\|u\|_{L^{2}\left(D_{\psi, \rho_{1}}^{T_{1}-\tau}\right)} \leq K \delta^{\vartheta_{1}}\|u\|_{L^{2}\left(D_{\psi, \rho_{2}}^{T_{1}}\right)}^{1-\vartheta_{1}}
$$

where

$$
\vartheta_{1}=\frac{\log \frac{\rho_{2}}{\rho_{1}}}{C \log \frac{C \rho_{2}}{\rho_{1}}}, \quad K=\gamma_{1}\left(\frac{C \rho_{2}}{\rho_{1}}\right)^{\gamma_{1}}, \quad \gamma_{1}=C\left(\frac{R^{2}}{T_{1}}+\frac{T_{1}}{\tau}\right)^{C} .
$$

PROOF. We present here only the most remarkable steps of the proof.

First of all, we extend the function $u$ to the cylinder $Q_{\psi, R}^{T_{1}}$ by setting it equal to zero outside $D_{\psi, R}^{T_{1}}$ and we continue to denote by $u$ such an extension. Then, let us introduce the change of variables $y=R^{-1} x, s=T_{1}^{-1} t$. Denoting by $v(y, s)=$ $u\left(R y, T_{1} s\right)$ and $h\left(y^{\prime}\right)=\psi\left(R y^{\prime}\right)$, we have that $v \in H^{1,1}\left(Q_{h, 1}^{1}\right) \cap H_{l o c}^{2,1}\left(Q_{h, 1}^{1}\right)$ is a solution to

$$
L v=\partial_{i}\left(g^{i j}(y, s) \partial_{j} v\right)-q_{0} \partial_{s} v=0 \text { in } Q_{h, 1}^{1}, \quad v=0 \text { on } \Gamma_{h, 1}^{1},
$$

where $g^{-1}(y, s)=\kappa\left(R y, T_{1} s\right)$ and $q_{0}=R^{2} T_{1}^{-1}$, and $v$ satisfies also

$$
\int_{\Gamma_{h, 1}^{1}}\left(g^{-1} \nabla v \mid \nu\right)^{2} \leq \delta^{2}
$$

Moreover, the matrix $g^{-1}$ satisfies (3.1) and (3.2) and $h$ is a function of Lipschitz class with constants $E, 1$ and such that $h(0)=0$.

Let $\varepsilon$ be a positive number to be chosen later. Let us denote

$$
w(y, s)=\varphi\left(\rho_{\varepsilon}(y, s)\right), \quad \text { where } \varphi(\eta)=\eta \exp \left(\int_{0}^{\eta} \frac{\mathrm{e}^{-\mu \tau}-1}{\tau} \mathrm{d} \tau\right)
$$

and

$$
\rho_{\varepsilon}(y, s)=\left(\sum_{i, j=1}^{N} g_{i j}(0, t)\left(y+\varepsilon e_{N}\right)_{i}\left(y+\varepsilon e_{N}\right)_{j}\right)^{1 / 2} .
$$

Proceeding in the same manner as in the proof of Theorem 3.5, see [21], it is not difficult to show that there exist constants $C>1, d \in(0,1)$, depending on $\lambda, \Lambda$ and $E$ only, such that, putting $\mu=C$ in (3.39), for any $\alpha \geq C\left(q_{0}+1\right)$ and 
any $\tilde{v} \in H^{1,1}\left(Q_{h, 1}^{1}\right) \cap H_{l o c}^{2,1}\left(Q_{h, 1}^{1}\right)$ such that $\tilde{v}=0$ on $\Gamma_{h, 1}^{1}$ and $\operatorname{supp} \tilde{v} \subset Q_{d}^{1, \rho_{\varepsilon}}$, the following inequality holds

$$
\begin{aligned}
C \int w^{2-2 \alpha}(L \tilde{v})^{2}-2 \alpha \int_{\Gamma_{h, 1}^{1}} \frac{w^{1-2 \alpha}(\nabla w \cdot \mathbf{n})}{|\nabla w|^{2}|\mathbf{n}|^{2}}(\nabla \tilde{v} \cdot \mathbf{n})^{2} \geq \\
\quad \geq \int\left(\alpha w^{1-2 \alpha}|\nabla \tilde{v}|^{2}+\alpha^{3} w^{-1-2 \alpha} \tilde{v}^{2}\right) .
\end{aligned}
$$

Let us apply the above stated inequality to the function $\tilde{v}=v \zeta$, where $v$ satisfies (3.37) and (3.38) and $\zeta$ is a function of class $C_{0}^{2}\left(Q_{d}^{1}\right)$ which is defined as follows. Let us fix $d_{1} \in(0, \lambda d), r \in\left(\varepsilon, d_{1} / 2\right)$ and $s_{0} \in(0,1)$. Let us denote $t_{1}=1-s_{0} / 2$, $t_{2}=1-s_{0}$. Let $\psi$ be the even function such that $\psi \in C_{0}^{2}(-1,1), \psi$ is equal to 1 in $\left[-t_{2}, t_{2}\right]$, it is equal to 0 in $\left[-1,-t_{1}\right]$ and

$$
\psi(s)=\exp \left(-\left(\frac{\left|t_{2}+s\right|}{t_{1}+s}\right)^{3}\right), \quad \text { for any } s \in\left(-t_{1},-t_{2}\right] .
$$

Let $f$ be a function in $C^{2}\left(\left[0, d_{1}\right]\right)$ that is equal to 1 in $\left[0, d_{1} / 2\right]$ and is equal to 0 in $\left[3 d_{1} / 4, d_{1}\right]$. Moreover, assume that $\left|f^{\prime}\right| \leq c / d_{1},\left|f^{\prime \prime}\right| \leq c / d_{1}^{2}$ in $\left[d_{1} / 2,3 d_{1} / 4\right]$, where $c$ is an absolute constant.

Then, $\zeta$ is defined by

$$
\zeta(y, s)=f\left(\rho_{\varepsilon}(y, s)\right) \psi(s),
$$

and we denote $Q_{h, r}^{t_{2}, \rho_{\varepsilon}}=\left\{(y, s) \in \mathbb{R}^{N+1}: \rho_{\varepsilon}(y, s)<r, y_{N}>h\left(y^{\prime}\right), s \in\right.$ $\left.\left(-t_{2}, t_{2}\right)\right\}$. Let us choose $\varepsilon=r \sqrt{\lambda} / 2$ in (3.39), (3.40). By (3.38) and (3.41) we have that there exists a constant $C>1$ depending on $\lambda, \Lambda$ and $E$ only such that for all $\alpha \geq C\left(q_{0} s_{0}^{-1}+1\right)^{12}$ the following inequality holds

$$
\|v\|_{L^{2}\left(Q_{h, r}^{t_{1}}\right)} \leq C\left(d_{1}^{-2}+q_{0} s_{0}^{-1}\right)\left(\left(\frac{\varphi(r)}{\varphi\left(d_{1} / 2\right)}\right)^{\alpha}\|u\|_{L^{2}\left(Q_{h, d_{1}}^{1}\right)}+\left(\frac{\varphi(r)}{\varphi\left(r_{0}\right)}\right)^{\alpha} \delta\right)
$$

where $r_{0}=\frac{r \sqrt{\lambda}}{2 \sqrt{1+E^{2}}}$.

Now, set

$$
\alpha_{1}=\frac{\log \left(\|u\|_{L^{2}\left(Q_{h, d_{1}}^{1}\right)} / \delta\right)}{\log \left(\varphi\left(d_{1} / 2\right) / \varphi\left(r_{0}\right)\right)} .
$$

If $\alpha_{1} \geq C\left(q_{0} s_{0}^{-1}+1\right)^{12}$ then we choose $\alpha=\alpha_{1}$ in (3.42) and we obtain

$$
\|v\|_{L^{2}\left(Q_{h, r}^{t_{1}}\right)} \leq C\left(d_{1}^{-2}+q_{0} s_{0}^{-1}\right) \delta^{\vartheta_{0}}\|u\|_{L^{2}\left(Q_{h, d_{1}}^{1}\right)}^{1-\vartheta_{0}},
$$

where

$$
\vartheta_{0}=\frac{\log \left(\varphi\left(d_{1} / 2\right) / \varphi(r)\right)}{\log \left(\varphi\left(d_{1} / 2\right) / \varphi\left(r_{0}\right)\right)} .
$$

If $\alpha_{1} \leq C\left(q_{0} s_{0}^{-1}+1\right)^{12}$ then, by an easy estimate from above of the left hand side of (3.42), we obtain

$$
\|v\|_{L^{2}\left(Q_{h, r}^{t_{1}}\right)} \leq C\left(d_{1}^{-2}+q_{0} s_{0}^{-1}+\left(C d_{1} / r\right)^{\alpha_{1}}\right) \delta^{\vartheta_{0}}\|u\|_{L^{2}\left(Q_{h, d_{1}}^{1}\right.}^{1-\vartheta_{0}} .
$$


By (3.43) and (3.44), returning to the original variables, it is easy to obtain the inequality (3.36).

In Proposition 3.7 below we shall use the following notation. Let $\alpha$ and $R$ be positive numbers such that $\alpha<\pi$ and let $s_{1} \in(0,1)$ be defined as in Theorem 3.4. Given $x_{0}, \zeta \in \mathbb{R}^{N}$, with $|\zeta|=1$, we denote

$$
\lambda_{1}=\frac{R}{1+\sin \alpha}, \quad w_{1}=x_{0}+\lambda_{1} \zeta, \quad \rho_{1}=(1 / 4) \lambda_{1} s_{1}^{2} \sin \alpha
$$

Furthermore, given a bounded domain $D$ in $\mathbb{R}^{N+1}$, a number $\beta, 0<\beta \leq 1$, and a function $u$ defined on $D$, we shall denote

$$
|u|_{\beta, \beta / 2, D}=\sup _{\substack{(x, t),(y, s) \in D \\(x, t) \neq(y, s)}} \frac{|u(x, t)-u(y, s)|}{\left(|x-y|^{2}+|t-s|\right)^{\beta / 2}} .
$$

Proposition 3.7 (Smallness Propagation Estimate) Let us take positive numbers $\alpha, \beta, H, T_{1}$ and $R$ such that $\alpha<\pi, T_{1} \in(0, T], R \in\left(0, R_{0}\right]$. Let $\kappa$ be a symmetric $N \times N$ matrix such that (2.7) and (2.8) are satisfied. Let $u \in H_{l o c}^{2,1}\left(C^{T_{1}}\left(x_{0}, \zeta, \alpha, R\right)\right)$ be a solution to

$$
\partial_{t} u-\operatorname{div}(\kappa(x, t) \nabla u)=0 \text { in } C^{T_{1}}\left(x_{0}, \zeta, \alpha, R\right), \quad u(x, 0)=0 \text { in } C\left(x_{0}, \zeta, \alpha, R\right),
$$

such that

$$
\|u\|_{L^{2}\left(C^{\left.T_{1}\left(x_{0}, \zeta, \alpha, R\right)\right)}\right.}+R^{\beta}|u|_{\beta, \beta / 2, C^{T_{1}\left(x_{0}, \zeta, \alpha, R\right)}} \leq H .
$$

Then, for every $t \in\left(0, T_{1} / 2\right)$,

$$
\left|u\left(x_{0}, t\right)\right| \leq C H\left|\log \left((\mathrm{e} H)^{-1}\|u\|_{L^{2}\left(D_{\rho_{1}}^{T_{1}}\left(w_{1}\right)\right)}\right)\right|^{-B},
$$

where $C$ depends on $\alpha, \beta, \lambda, \Lambda$ and $R^{2} / T_{1}$ only and $B$ depends on $\alpha, \beta, \lambda$ and $\Lambda$ only.

ProOF. For the sake of simplicity we assume that $x_{0}=0, \zeta=e_{N}$. Let

$$
a=\frac{1-(1 / 4) s_{1}^{2} \sin \alpha}{1+(1 / 4) s_{1}^{2} \sin \alpha}
$$

and, for every $k \geq 2$,

$$
\lambda_{k}=a^{k-1} \lambda_{1}, \quad w_{k}=\lambda_{k} e_{N}, \quad \rho_{k}=a^{k-1} \rho_{1} .
$$

It is simple to check that, for every $k \geq 1$, the following inclusions hold true

$$
B_{\rho_{k+1}}\left(w_{k+1}\right) \subset B_{3 \rho_{k}}\left(w_{k}\right) \subset B_{4 s_{1}^{-2} \rho_{k}}\left(w_{k}\right) \subset C\left(0, e_{N}, \alpha, R\right) .
$$

Denote by

$$
d_{k}=\lambda_{k}-\rho_{k}=a^{k-1} \lambda_{1}\left(1-(1 / 4) s_{1}^{2} \sin \alpha\right), \quad k \geq 1 .
$$

For a number $r$ belonging to $\left(0, d_{1}\right]$, to be chosen later, let us set $\bar{k}=\min \{k \in$ $\left.\mathbb{N}: d_{k} \leq r\right\}$. We have

$$
\frac{\left|\log \left(r / d_{1}\right)\right|}{|\log a|} \leq \bar{k}-1 \leq \frac{\left|\log \left(r / d_{1}\right)\right|}{|\log a|}+1 .
$$


Moreover, for $j=0,1, \ldots, \bar{k}$, we set $t_{j}=T_{1}\left(1-\frac{j}{2 \bar{k}}\right)$ and $\sigma_{j}=\|u\|_{L^{2}\left(D_{\rho_{j}}^{t_{j}}\left(w_{j}\right)\right)}$.

By Theorem 3.4 and (3.48), and since obviously $\sigma_{j+1} \leq\|u\|_{L^{2}\left(D_{3 \rho_{j+1}}^{t_{j}+1}\left(w_{j+1}\right)\right)}$, we obtain

$$
\sigma_{j+1} \leq \bar{K} \sigma_{j}^{\theta_{*}}\left(\|u\|_{L^{2}\left(C^{\left.T_{1}\left(0, e_{N}, \alpha, R\right)\right)}\right.}\right)^{1-\theta_{*}}, \quad \text { for } j=0,1, \ldots, \bar{k},
$$

where

$$
\theta_{*}=\frac{\log \left(4 / 3 s_{1}\right)}{C_{1} \log \left(4 C_{1} / s_{1}^{2}\right)}, \quad \bar{K}=\mathrm{e}^{C_{2}(1+\bar{k})^{C_{1}}},
$$

and here $C_{1}$ depends on $\lambda$ and $\Lambda$ only and $C_{2}$ depends on $\lambda, \Lambda$ and $R^{2} / T_{1}$ only.

By iterating (3.50), we obtain that

$$
\sigma_{\bar{k}} \leq \bar{K}^{1 /\left(1-\theta_{*}\right)} \sigma_{1}^{\theta_{*}^{\bar{k}}}\left(\|u\|_{L^{2}\left(C^{T_{1}}\left(0, e_{N}, \alpha, R\right)\right)}\right)^{1-\theta_{*}^{\bar{k}}} .
$$

Let us recall the following interpolation inequality

$$
\begin{aligned}
&\|v\|_{L^{\infty}\left(D_{\rho}^{T_{0}}\right)} \leq C\left(\rho_{0}^{-(N+2)} \int_{D_{\rho}^{T_{0}}} v^{2}\right)^{\frac{\beta}{N+2+2 \beta}}\left(|v|_{\beta, \beta / 2, D_{\rho}^{T_{0}}}\right)^{\frac{N+2}{N+2+2 \beta}} \\
&+C\left(\rho_{0}^{-(N+2)} \int_{D_{\rho}^{T_{0}}} v^{2}\right)^{1 / 2},
\end{aligned}
$$

where $\rho_{0}=\min \left\{\rho, \sqrt{T_{0}}\right\}$ and $C$ is an absolute constant.

By (3.46), (3.51) and (3.52), we obtain

$$
\|u\|_{L^{\infty}\left(D_{\rho_{\bar{k}}}^{t}\left(w_{\bar{k}}\right)\right)} \leq C\left(a^{1-\bar{k}}\right)^{(N+2) / 2} H^{\frac{N+2}{N+2+2 \beta}}\left(\sigma_{\bar{k}}\right)^{\frac{2 \beta}{N+2+2 \beta}},
$$

where $C$ depends on $\lambda, \Lambda$ and $R^{2} / T_{1}$ only. Let us consider the point $x_{r}=r e_{N}$. We have that $\left(x_{r}, t\right) \in D_{\rho_{\bar{k}}}^{t_{\bar{k}}}\left(w_{\bar{k}}\right)$ for every $t \in\left(0, T_{1} / 2\right)$. By (3.46) and (3.53), we have

$$
\begin{aligned}
& |u(0, t)| \leq\left|u\left(x_{r}, t\right)-u(0, t)\right|+\left|u\left(x_{r}, t\right)\right| \leq \\
& \quad \leq C H\left(\left(\frac{r}{d_{1}}\right)^{\beta}+\left(a^{1-\bar{k}}\right)^{(N+2) / 2}\left(\frac{\sigma_{\bar{k}}}{H}\right)^{\frac{2 \beta}{N+2+2 \beta}}\right),
\end{aligned}
$$

where $C$ depends on $\lambda, \Lambda$ and $R^{2} / T_{1}$ only.

From this last inequality and (3.51) we obtain

$$
|u(0, t)| \leq C H\left(\left(\frac{r}{d_{1}}\right)^{\beta}+\left(a^{1-\bar{k}}\right)^{(N+2) / 2} \mathrm{e}^{C(1+\bar{k})^{C_{1}}}\left(\frac{\sigma_{1}}{H}\right)^{\frac{2 \beta \theta_{\bar{K}}}{N+2+2 \beta}}\right),
$$

where $C$ depends on $\alpha, \beta, \lambda, \Lambda$ and $R^{2} / T_{1}$ only and $C_{1}$ depends on $\lambda$ and $\Lambda$ only. Let us choose

$$
r=d_{1}\left|\log \left(\frac{1}{\mathrm{e}}\left(\frac{\sigma_{1}}{H}\right)^{\frac{2 \beta}{N+2+2 \beta}}\right)\right|^{-\frac{|\log a|}{2\left|\log \theta_{*}\right|}} .
$$

From (3.54), taking into account (3.49), we obtain

$$
|u(0, t)| \leq C H\left|\log \left(\frac{\sigma_{1}}{\mathrm{e} H}\right)\right|^{-\frac{|\log a|}{2 \beta\left|\log \theta_{*}\right|}}
$$

and (3.47) follows. 


\section{Proof of Theorem 2.4}

Given the results obtained in the previous section, the proof of Theorem 2.4 can be concluded with a procedure which is analogous to the one used to prove the main theorems of $[4,5]$. However, for the convenience of the reader, we point out in this section the most important steps of the proof. We begin by stating the following propositions. In this section we shall denote by $G$ the connected component of $\Omega_{1} \cap \Omega_{2}$ such that $\Sigma \subset \bar{G}$.

Proposition 4.1 Let the hypotheses of Theorem 2.4 be satisfied. We have

$$
\max _{\left(\bar{\Omega}_{i} \backslash G\right) \times[0, T / 2]}\left|u_{i}\right| \leq\|f\| \omega(\varepsilon /\|f\|),
$$

where $\omega$ is an increasing continuous function on $[0, \infty)$ satisfying

$$
\omega(t) \leq C(\log |\log t|)^{-1 / N}, \quad \text { for any } 0<t<\mathrm{e}^{-1},
$$

and $C$ depends on the a priori data only.

Furthermore, if in addition we assume that there exist $L>0$ and $r_{0} \in\left(0, R_{0}\right]$ such that $\partial G$ is of Lipschitz class with constants $r_{0}, L$, then (4.1) holds true with $\omega$ satisfying

$$
\omega(t) \leq C_{1}|\log t|^{-C_{2}}, \quad \text { for any } 0<t<1,
$$

where $C_{1}, C_{2}$ depend on $R_{0} / r_{0}$ and the a priori data only.

Proposition 4.2 Let $\Omega$ be a bounded domain in $\mathbb{R}^{N}$ satisfying (2.1) and (2.2). Let $u \in W(\Omega \times(0, T))$ be the solution to (1.1), where $f$ satisfies (2.6) and $\kappa$ satisfies (2.7), (2.8). For every $\rho>0$ and every $x_{0} \in \Omega_{\rho}=\{x \in \Omega: \operatorname{dist}(x, \partial \Omega)>$ $\rho\}$, we have

$$
\|u\|_{L^{2}\left(D_{\rho}^{T / 4}\left(x_{0}\right)\right)} \geq C\|f\|,
$$

where $C$ depends on $R_{0} / \rho$ and the a priori data only.

Up to obvious changes, Proposition 4.1 can be proved following the lines of the proof of Proposition 5.2 in [5] and using standard regularity estimate for parabolic equations, see for instance [15, Chapter VI, Section 11], whereas the proof of Proposition 4.2 is similar to that of Proposition 5.5 in [4]. Here we recall the definition of modified distance introduced in [1].

Definition 4.3 We call modified distance between $\Omega_{1}$ and $\Omega_{2}$ the number

$$
d_{m}\left(\Omega_{1}, \Omega_{2}\right)=\max \left\{\sup _{x \in \partial \Omega_{1}} \operatorname{dist}\left(x, \bar{\Omega}_{2}\right), \sup _{x \in \partial \Omega_{2}} \operatorname{dist}\left(x, \bar{\Omega}_{1}\right)\right\} .
$$

Note that

$$
d_{m}\left(\Omega_{1}, \Omega_{2}\right) \leq d_{\mathcal{H}}\left(\bar{\Omega}_{1}, \bar{\Omega}_{2}\right)
$$

but, in general, the reverse inequality does not hold. However, the following proposition holds true, [1]. 
Proposition 4.4 Let $\Omega_{1}$ and $\Omega_{2}$ be bounded domains satisfying (2.1) and (2.2). For any $i=1,2$, let $A_{i}, I_{i}$ satisfy (2.3). Let us also assume that $A_{1}=A_{2}=A$ and that $\Omega_{1}$ and $\Omega_{2}$ lie on the same side of $A$. There exist numbers $d_{0}>0$, $r_{0} \in\left(0, R_{0}\right]$, such that $\frac{d_{0}}{R_{0}}$ and $\frac{r_{0}}{R_{0}}$ depend on $E$ only and the following facts hold true. If

$$
d_{\mathcal{H}}\left(\bar{\Omega}_{1}, \bar{\Omega}_{2}\right) \leq d_{0}
$$

then there exists an absolute constant $C>0$ such that

$$
d_{\mathcal{H}}\left(\bar{\Omega}_{1}, \bar{\Omega}_{2}\right) \leq C d_{m}\left(\Omega_{1}, \Omega_{2}\right),
$$

and any connected component of $\Omega_{1} \cap \Omega_{2}$ has boundary of Lipschitz class with constants $r_{0}, L$ where $r_{0}$ is as above and $L>0$ depends on $E$ only.

Proof of TheOREM 2.4. For the sake of simplicity we denote $d=d_{\mathcal{H}}\left(\bar{\Omega}_{1}, \bar{\Omega}_{2}\right)$ and $d_{m}=d_{m}\left(\Omega_{1}, \Omega_{2}\right)$. Let us prove that if $\sigma>0$ is such that

$$
\left\|u_{i}\right\|_{L^{2}\left(\left(\Omega_{i} \backslash G\right) \times(0, T / 2)\right)} \leq \sigma, \quad i=1,2,
$$

then

$$
d, d_{m} \leq C_{1} R_{0}(\sigma /\|f\|)^{C_{2}}
$$

where $C_{1}, C_{2}$ depend on the a priori data only.

We begin by proving (4.10) for $d_{m}$. We may assume, without loss of generality, that there exists $x_{0} \in I_{1} \subset \partial \Omega_{1}$ such that $\operatorname{dist}\left(x_{0}, \Omega_{2}\right)=d_{m}$. By (4.9) we have

$$
\left\|u_{1}\right\|_{L^{2}\left(\left(\Omega_{i} \cap B_{d_{m}}\left(x_{0}\right)\right) \times(0, T / 2)\right)} \leq \sigma .
$$

Let $s_{1}$ be defined as in Theorem 3.5. We distinguish two cases. If $d_{m} \geq s_{1}^{2} R_{0} / 2$, let $\bar{d}=\frac{s_{1}^{2} R_{0}}{2\left(1+\sqrt{1+E^{2}}\right)}, \bar{x}=x_{0}-\nu \bar{d} \sqrt{1+E^{2}}$, where $\nu$ denotes the outer unit normal to $\Omega_{1}$ at $x_{0}$. We have

$$
B_{\bar{d}}(\bar{x}) \subset \Omega_{1} \cap B_{\left(s_{1}^{2} R_{0} / 2\right)}\left(x_{0}\right) .
$$

By Proposition 4.2, (4.11) and the above inclusion we have

$$
\sigma \geq\left\|u_{1}\right\|_{L^{2}\left(\left(\Omega_{i} \cap B_{s_{1}^{2} R_{0} / 2}\left(x_{0}\right)\right) \times(0, T / 2)\right)} \geq C\|f\|,
$$

where $C$ depends on the a priori data only. Since it is evident that $d_{m} \leq C R_{0}$, where $C$ depends on $E$ and $M$ only, and $d_{m} \geq s_{1}^{2} R_{0} / 2$, by (4.12) we have that, in this case, $d_{m}$ satisfies (4.10) with $C_{1}$ depending on the a priori data only and $C_{2}=1$.

Otherwise, if $d_{m}<s_{1}^{2} R_{0} / 2$, let us apply Theorem 3.5 with $\rho_{1}=d_{m}, \rho_{2}=$ $s_{1}^{2} R_{0} / 2, R=R_{0}, T_{1}=T / 2, \tau=T / 4$ and, by (4.11), we obtain

$$
\left\|u_{1}\right\|_{L^{2}\left(\left(\Omega_{1} \cap B_{s_{1}^{2} R_{0} / 2}\left(x_{0}\right)\right) \times(0, T / 4)\right)} \leq C\|f\|(\sigma /\|f\|)^{\frac{1}{C \log \frac{C R_{0}}{d_{m}}}},
$$

where $C$ depends on the a priori data only. Then, by Proposition 4.2 and the inequality above, we can conclude that $d_{m}$ satisfies (4.10) also in this case. 
We have proved that (4.10) holds for $d_{m}$ and, now, we use this result to show that (4.10) holds for $d$, too. Without loss of generality, we may assume that there exists $y_{0} \in \bar{\Omega}_{1}$ such that $\operatorname{dist}\left(y_{0}, \bar{\Omega}_{2}\right)=d$. Let us denote $\delta=\operatorname{dist}\left(y_{0}, \partial \Omega_{1}\right)$ and let us distinguish three cases, depending on the value of $\delta$ with respect to $d$ and $d_{0}, d_{0}$ as in Proposition 4.4

First, if $\delta \leq d / 2$, we take $z_{0} \in \partial \Omega_{1}$ such that $\left|y_{0}-z_{0}\right|=\delta$ and we have

$$
d_{m} \geq \operatorname{dist}\left(z_{0}, \bar{\Omega}_{2}\right) \geq d-\delta \geq \delta / 2
$$

hence $\delta \leq 2 d_{m}$ and, since (4.10) holds for $d_{m}$, we have that it also holds for $d$.

Second, if $d / 2<\delta \leq d_{0} / 2$, then $d<d_{0}$ and Proposition 4.4 applies, hence by (4.8) $d$ can be controlled by $d_{m}$ and therefore $d$ satisfies (4.10) as well.

Third, if $\delta>\max \left\{d / 2, d_{0} / 2\right\}$, let us set $d_{1}=\min \left\{d / 2, s_{1}^{2} d_{0} / 4\right\}$ where $s_{1} \in$ $(0,1)$ has been introduced in Theorem 3.4. We have

$$
B_{d_{1}}\left(y_{0}\right) \subset \Omega_{1} \backslash \Omega_{2}, \quad B_{s_{1}^{2} d_{0} / 2}\left(y_{0}\right) \subset \Omega_{1} .
$$

Now, let us apply Theorem 3.4 with $\rho_{1}=d_{1}, \rho_{2}=s_{1}^{2} d_{0} / 2, R=d_{0}, T_{1}=T / 2$, $\tau=T / 4$ and, by (4.9) and (4.13), we have

$$
\left\|u_{1}\right\|_{L^{2}\left(\left(\Omega_{1} \cap B_{s_{1}^{2} d_{0} / 2}\left(y_{0}\right)\right) \times(0, T / 4)\right)} \leq C\|f\|(\sigma /\|f\|)^{\frac{1}{C \log \frac{C R_{0}}{d_{m}}}}
$$

where $C$ depends on the a priori data only. Then, by Proposition 4.2, we obtain that

$$
d_{1} \leq C_{1} R_{0}(\sigma /\|f\|)^{C_{2}}
$$

with $C_{1}$ and $C_{2}$ depending on the a priori data only.

Now, let $\tilde{\sigma}=\|f\|\left(\frac{s_{1}^{2} d_{0}}{4 C_{1} R_{0}}\right)^{C_{2}}$. If $\sigma<\tilde{\sigma}$, then $d_{1}<\left(s_{1}^{2} d_{0}\right) / 4$, hence $d=2 d_{1}$ and (4.10) follows by (4.14). If $\sigma \geq \tilde{\sigma}$ then (4.10) immediately follows.

By Proposition 4.1 and (4.10) we have

$$
d \leq\left. C_{1} R_{0}|\log | \log (\varepsilon /\|f\|)\right|^{-C_{2}}
$$

where $C_{1}, C_{2}$ depend on the a priori data only. Then, there exists $\varepsilon_{0}>0$, depending on the a priori data only, such that if $\varepsilon \leq \varepsilon_{0}$ then $d \leq d_{0}$. Thus, by Proposition 4.4, $G$ satisfies the hypotheses of Proposition 4.2. Hence in (4.10) we may replace $\sigma$ with $\|f\| \omega(\varepsilon /\|f\|)$ where $\omega(t) \leq C_{1}|\log t|^{-C_{2}}, C_{1}, C_{2}$ depending on the a priori data only, and thus (2.10) follows.

\section{Proof of Theorem 2.5}

We begin with the following definition.

Definition 5.1 Let $(X, d)$ be a metric space. For a given positive $\delta, Z \subset X$ is $\delta$-discrete if for any two distinct points $z_{1}, z_{1}^{\prime}$ in $Z$ we have $d\left(z_{1}, z_{1}^{\prime}\right) \geq \delta$.

Let $(X, d)$ be as in the hypotheses of Theorem 2.5. Let us set $D_{0}=\bar{B}_{1 / 2}$ and let us call, for any $\delta>0, X_{\delta}=\left\{D \in X: d\left(D, D_{0}\right) \leq \delta\right\}$. We observe that for any $\delta, 0<\delta \leq 1 / 4$, we have $X_{\delta}=X_{m b \delta}\left(\bar{B}_{1 / 2}\right)$. We have that $(X, d)$ satisfies the following proposition, see for instance [7, Proposition 3.1]. 
Proposition 5.2 There exists $\delta_{0}>0$, depending on $m$ and $b$ only, such that for any $\delta, 0<\delta<\delta_{0}$, we can find $Z_{\delta} \subset X_{\delta}$ such that $Z_{\delta}$ is $\delta$-discrete, with respect to the Hausdorff distance, and $Z_{\delta}$ has at least $\exp \left(2^{-N} \delta_{0}^{(N-1) / m} \delta^{-(N-1) / m}\right)$ elements.

Before proving Theorem 2.5, we need to introduce some further notation. Let $\tilde{Q}=\Omega \times(0,2 \pi), \tilde{\Gamma}=A \times(0,2 \pi)$ and, for any $D \in X$, let $\tilde{Q}(D)=(\Omega \backslash D) \times(0,2 \pi)$ and $\tilde{\Gamma}(D)=\partial D \times(0,2 \pi)$. Clearly, we set $\tilde{Q}(\emptyset)=\tilde{Q}$ and $\tilde{\Gamma}(\emptyset)=\emptyset$.

Let us consider the Hilbert space $L^{2}(\tilde{\Gamma})$ endowed with the scalar product

$$
(\psi, \phi)_{0}=\int_{\tilde{\Gamma}} \psi \phi, \quad \text { for any } \psi, \phi \in L^{2}(\tilde{\Gamma}) .
$$

We can choose as an orthonormal basis of $L^{2}(A)$ the following set

$$
\left\{f_{j p}: j \geq 0 \text { and } 1 \leq p \leq p_{j}\right\}
$$

where each $f_{j p}$ is a real valued spherical harmonic of degree $j, j$ being a nonnegative integer. Therefore, we have that

$$
-\Delta_{A} f_{j p}=\lambda_{j} f_{j p}=j(j+N-2) f_{j p},
$$

where $\Delta_{A}$ is the Laplace-Beltrami operator on $A$. For any $j \geq 0$, the integer $p_{j}$ is the dimension of the space of spherical harmonics of degree $j$ and we have that, see for instance [19, page 4],

$$
p_{j}= \begin{cases}1 & \text { if } j=0 \\ \frac{(2 j+N-2)(j+N-3) !}{j !(N-2) !} & \text { if } j \geq 1,\end{cases}
$$

so that

$$
p_{j} \leq 2(j+1)^{N-2}, \quad j \geq 0 .
$$

Then we have that the set

$$
\left\{\psi_{n j p}=\frac{1}{\sqrt{\pi}} \sin \left(\frac{n}{2} t\right) f_{j p}(\omega): n \geq 1, j \geq 0 \text { and } 1 \leq p \leq p_{j}\right\}
$$

is an orthonormal basis of $L^{2}(\tilde{\Gamma})$ with respect to the scalar product $(\cdot, \cdot)_{0}$.

For any $s, 0<s \leq 1$, let us consider the Sobolev space $H_{, 0}^{2 s, s}(\tilde{\Gamma})$. We have the following properties. First, $H_{, 0}^{2 s, s}(\tilde{\Gamma})=H^{2 s, s}(\tilde{\Gamma})$ if and only if $s \leq 1 / 2$. Then, for any $s \neq 1 / 2$, we can endow $H_{, 0}^{2 s, s}(\tilde{\Gamma})$ with the following scalar product

$(\psi, \phi)_{s}=\sum_{\substack{n \geq 1, j \geq 0 \\ 1 \leq p \leq p_{j}}}\left(1+\lambda_{j}^{2 s}+\left(\frac{n}{2}\right)^{2 s}\right)\left(\psi, \psi_{n j p}\right)_{0}\left(\phi, \psi_{n j p}\right)_{0}, \quad$ for any $\psi, \phi \in H_{, 0}^{2 s, s}(\tilde{\Gamma})$,

with respect to which the set

$$
\left\{\tilde{\psi}_{n j p}=\frac{\psi_{n j p}}{\sqrt{1+\lambda_{j}^{2 s}+\left(\frac{n}{2}\right)^{2 s}}}: n \geq 1, j \geq 0 \text { and } 1 \leq p \leq p_{j}\right\}
$$

is an orthonormal basis of $H_{, 0}^{2 s, s}(\tilde{\Gamma})$. 
For any $n, j$ and $p$, let us define $\gamma\left(\tilde{\psi}_{n j p}\right)=\sqrt{n+j}$ and let us call, for any positive integer $q, N_{1}(q)=\#\left\{(n, j, p): \gamma\left(\tilde{\psi}_{n j p}\right) \leq q\right\}$, where \# denotes the number of elements. By the previous estimate on $p_{j}$, we have that

$$
N_{1}(q) \leq 2(1+q)^{2 N}, \quad \text { for any } q \in \mathbb{N} .
$$

Let $\tilde{H}=H_{, 0}^{3 / 2,3 / 4}(\tilde{\Gamma}), \tilde{H}^{\prime}=\tilde{H}_{1}=H^{-3 / 2,-3 / 4}(\tilde{\Gamma})$ and $\tilde{H}_{0}=H^{1 / 2,1 / 4}(\tilde{\Gamma})$. For any $D \in X \cup\{\emptyset\}$, let us define in the same fashion as before the Dirichletto-Neumann map associated to $D$, that is the linear and bounded operator $\tilde{\mathcal{D}}(D): \tilde{H} \mapsto \tilde{H}_{0}$ such that for any $\tilde{\psi} \in \tilde{H}$ we have

$$
\tilde{\mathcal{D}}(D) \tilde{\psi}=\left.\frac{\partial \tilde{u}}{\partial \nu}\right|_{\Gamma},
$$

where $\tilde{u}$ solves

$$
\begin{cases}\partial_{t} \tilde{u}-\Delta \tilde{u}=0 & \text { in } \tilde{Q}(D), \\ \tilde{u}(x, 0)=0 & x \in \Omega \backslash D, \\ \tilde{u}=0 & \text { on } \tilde{\Gamma}(D), \\ \tilde{u}=\tilde{\psi} & \text { on } \tilde{\Gamma} .\end{cases}
$$

Let us also define the following two linear and bounded operators $\tilde{G}, \tilde{G}^{*}$ : $\tilde{H} \mapsto \tilde{H}$ such that for any $\tilde{\psi} \in \tilde{H}$ we have

$$
\begin{aligned}
& \tilde{G} \tilde{\psi}(\omega, t)=\tilde{\psi}(\omega, t) \mathrm{e}^{-1 / t} t^{-3 / 2}, \\
& \tilde{G}^{*} \tilde{\psi}(\omega, t)=\tilde{\psi}(\omega, t) \mathrm{e}^{-1 /(2 \pi-t)}(2 \pi-t)^{-3 / 2} .
\end{aligned}
$$

We have that there exists a constant $C_{1}$ such that

$$
\|\tilde{G}\|_{\mathcal{L}(\tilde{H}, \tilde{H})},\left\|\tilde{G}^{*}\right\|_{\mathcal{L}(\tilde{H}, \tilde{H})} \leq C_{1} .
$$

Now we can define, for any $D \in X \cup\{\emptyset\}$, the following linear and bounded operator $\tilde{\mathcal{D}}_{1}(D): \tilde{H} \mapsto \tilde{H}^{\prime}$ as follows

$$
\left\langle\tilde{\mathcal{D}}_{1}(D) \tilde{\psi}, \tilde{\phi}\right\rangle_{\tilde{H}^{\prime}, \tilde{H}}=\left\langle\tilde{\mathcal{D}}(D) \tilde{G} \tilde{\psi}, \tilde{G}^{*} \tilde{\phi}\right\rangle_{\tilde{H}^{\prime}, \tilde{H}}, \quad \text { for any } \tilde{\psi}, \tilde{\phi} \in \tilde{H} .
$$

Then the proof of Theorem 2.5 is an immediate consequence of the following two propositions.

Proposition 5.3 There exists a constant $C_{2}$, depending on $m$ and $b$ only, such that for any $D \in X$ and any $\tilde{\psi} \in \tilde{H}$

$$
\|(\tilde{\mathcal{D}}(D)-\tilde{\mathcal{D}}(\emptyset)) \tilde{\psi}\|_{\tilde{H}_{0}} \leq C_{2}\left\|\tilde{u}_{0}\right\|_{H^{2,1}\left(B_{7 / 8} \times(0,2 \pi)\right)},
$$

where $\tilde{u}_{0}$ solves (5.7) with $D=\emptyset$.

Proposition 5.4 There exists a positive constant $\delta_{1}$, depending on $m$ and $b$ only, such that for any $\delta, 0<\delta<\delta_{1}$, we can find $D_{1}, D_{2} \in X$ satisfying (2.17) and such that

$$
\left\|\tilde{\mathcal{D}}_{1}\left(D_{1}\right)-\tilde{\mathcal{D}}_{1}\left(D_{2}\right)\right\|_{\mathcal{L}\left(\tilde{H}, \tilde{H}^{\prime}\right)} \leq 2 \exp \left(-\delta^{-\frac{N-1}{2 m(2 N+1)}}\right) .
$$


Proof of Theorem 2.5. We observe that there exists a constant $C_{3}$ such that the following two inequalities are satisfied for any $\tilde{\psi} \in \tilde{H}$

$$
\|\tilde{\mathcal{D}}(\emptyset) \tilde{\psi}\|_{\tilde{H}_{0}} \leq C_{3}\left\|\tilde{u}_{0}\right\|_{H^{2,1}(\tilde{Q})}, \quad\left\|\tilde{u}_{0}\right\|_{H^{2,1}(\tilde{Q})} \leq C_{3}\|\tilde{\psi}\|_{\tilde{H}} .
$$

By Proposition 5.3, we have that there exists a constant $C_{4}$, depending on $m$ and $b$ only, such that

$$
\|\tilde{\mathcal{D}}(D)\|_{\mathcal{L}\left(\tilde{H}, \tilde{H}_{0}\right)} \leq C_{4}, \quad \text { for any } D \in X .
$$

Then, we consider the following fact. For any $\psi \in H$, let $\tilde{\psi} \in \tilde{H}$ be its extension by 0 outside $\Gamma$. We have that $J: H \mapsto \tilde{H}$, where $J(\psi)=\tilde{\psi}$ for any $\psi \in H$, is a linear isometry. Furthermore, we have that the linear operators $G, G^{*}: H \mapsto H$ such that for any $\psi \in H$

$$
G(\psi)=\left.\tilde{G}(\tilde{\psi})\right|_{\Gamma}, \quad G^{*}(\psi)=\left.\tilde{G}^{*}(\tilde{\psi})\right|_{\Gamma},
$$

are invertible and there exists a constant $C_{5}$ such that

$$
\|G\|_{\mathcal{L}(H, H)},\left\|G^{*}\right\|_{\mathcal{L}(H, H)},\left\|G^{-1}\right\|_{\mathcal{L}(H, H)},\left\|\left(G^{*}\right)^{-1}\right\|_{\mathcal{L}(H, H)} \leq C_{5} .
$$

For any $D \in X$, for any $\psi, \phi \in H$, we have that

$$
\mathcal{D}(D) \psi=\left.\tilde{\mathcal{D}}(D) J \psi\right|_{\Gamma}
$$

and

$$
\langle\mathcal{D}(D) \psi, \phi\rangle_{H^{\prime}, H}=\langle\tilde{\mathcal{D}}(D) J \psi, J \phi\rangle_{\tilde{H}^{\prime}, \tilde{H}}=\left\langle\tilde{\mathcal{D}}_{1}(D) J G^{-1} \psi, J\left(G^{*}\right)^{-1} \phi\right\rangle_{\tilde{H}^{\prime}, \tilde{H}} .
$$

By (5.15) and (5.13), we infer that for any $D_{1}, D_{2} \in X$ we have

$$
\left\|\mathcal{D}\left(D_{1}\right)-\mathcal{D}\left(D_{2}\right)\right\|_{\mathcal{L}\left(H, H_{0}\right)} \leq 2 C_{4} .
$$

By (5.16) and (5.14), we infer that for any $D_{1}, D_{2} \in X$ we have

$$
\left\|\mathcal{D}\left(D_{1}\right)-\mathcal{D}\left(D_{2}\right)\right\|_{\mathcal{L}\left(H, H^{\prime}\right)} \leq C_{5}^{2}\left\|\tilde{\mathcal{D}}_{1}\left(D_{1}\right)-\tilde{\mathcal{D}}_{1}\left(D_{2}\right)\right\|_{\mathcal{L}\left(\tilde{H}, \tilde{H}^{\prime}\right)} .
$$

The proof of Theorem 2.5 follows immediately from Proposition 5.4, (5.17), (5.18) and the interpolation inequality (2.12).

Proof of Proposition 5.3. Let $\chi \in C_{0}^{\infty}\left(B_{1}\right)$ be a cutoff function such that

$$
0 \leq \chi \leq 1, \quad \chi \equiv 1 \text { in } B_{5 / 6}, \quad \chi \equiv 0 \text { outside } B_{7 / 8} .
$$

Let us take the auxiliary function $v=\tilde{u}-(1-\chi) \tilde{u}_{0}, \tilde{u}$ being the solution to (5.7). We have that $\left.\frac{\partial v}{\partial \nu}\right|_{\tilde{\Gamma}}=(\tilde{\mathcal{D}}(D)-\tilde{\mathcal{D}}(\emptyset)) \tilde{\psi}$ and that $v$ solves

$$
\begin{cases}\partial_{t} v-\Delta v=f & \text { in } \tilde{Q}(D), \\ v(x, 0)=0 & x \in \Omega \backslash D, \\ v=0 & \text { on } \tilde{\Gamma}(D), \\ v=0 & \text { on } \tilde{\Gamma},\end{cases}
$$


where $f=-\Delta \chi \tilde{u}_{0}-2 \nabla \chi \cdot \nabla \tilde{u}_{0}$ and hence, by construction of $\chi$, it is supported in $\left(\bar{B}_{7 / 8} \backslash B_{5 / 6}\right) \times[0,2 \pi]$ and, for a constant $C_{6}$,

$$
\|f\|_{L^{2}\left(\tilde{Q}\left(\bar{B}_{1 / 2}\right)\right.} \leq C_{6}\left\|\tilde{u}_{0}\right\|_{H^{2,1}\left(B_{7 / 8} \times(0,2 \pi)\right)} .
$$

Then we perform the following change of variables. There exists a constant $C_{7}$, depending on $m$ and $b$ only, such that for any $D \in X$ we can find a bijective function $\varphi: B_{1} \mapsto B_{1}$ satisfying

$$
\begin{aligned}
& \varphi(x)=x, \quad \text { for any } x \in \bar{B}_{1 / 4} \cup\left(B_{1} \backslash B_{5 / 6}\right), \quad \varphi\left(\bar{B}_{1 / 2}\right)=D, \\
& \|\varphi\|_{C^{m}\left(\bar{B}_{1}\right)},\left\|\varphi^{-1}\right\|_{C^{m}\left(\bar{B}_{1}\right)} \leq C_{7} .
\end{aligned}
$$

Let $w(x, t)=v(\varphi(x), t)$. We have that $w$ solves

$$
\begin{cases}a \partial_{t} w-\operatorname{div}(A \nabla w)=f & \text { in } \tilde{Q}\left(\bar{B}_{1 / 2}\right), \\ w(x, 0)=0 & x \in \Omega \backslash B_{1 / 2}, \\ w=0 & \text { on } \tilde{\Gamma}\left(\bar{B}_{1 / 2}\right), \\ w=0 & \text { on } \Gamma,\end{cases}
$$

where $a$ is a function depending on $x$ only, $A$ is a symmetric $N \times N$ matrix depending on $x$ only, and there exists a constant $c_{1}>0$, depending on $m$ and $b$ only, such that for any $x \in B_{1}$ we have

$$
\begin{aligned}
& a(x) \geq c_{1}, \quad A(x) \Xi \cdot \Xi \geq c_{1}\|\Xi\|^{2}, \text { for any } \Xi \in \mathbb{R}^{N}, \\
& \|a\|_{C^{m-1}\left(\bar{B}_{1}\right)},\|A\|_{C^{m-1}\left(\bar{B}_{1}\right)} \leq 1 / c_{1} .
\end{aligned}
$$

By standard regularity estimates, see for instance [13, Chapter III, Section 6], we obtain that there exists a constant $C_{8}$, depending on $c_{1}$ only, so that

$$
\|w\|_{H^{2,1}\left(\tilde{Q}\left(\bar{B}_{1 / 2}\right)\right)} \leq C_{8}\|f\|_{L^{2}\left(\tilde{Q}\left(\bar{B}_{1 / 2}\right)\right)} .
$$

Therefore the proof is concluded by noticing that, by the trace theorem $[16$, Chapter 4, Theorem 2.1], there exists a constant $C_{9}$ such that

$$
\left\|\left.\frac{\partial w}{\partial \nu}\right|_{\tilde{\Gamma}}\right\|_{\tilde{H}_{0}} \leq C_{9}\|w\|_{H^{2,1}\left(\tilde{Q}\left(\bar{B}_{1 / 2}\right)\right)}
$$

and that, by construction, $\left.\frac{\partial w}{\partial \nu}\right|_{\tilde{\Gamma}}=\left.\frac{\partial v}{\partial \nu}\right|_{\tilde{\Gamma}}$.

We now turn our attention to Proposition 5.4. Let us fix integers $n \geq 1$, $j \geq 0, p, 1 \leq p \leq p_{j}$, and let

$$
\Psi_{n j p}(\omega, t)=y_{n}(t) f_{j p}(\omega), \quad(\omega, t) \in A \times \mathbb{R},
$$

where

$$
y_{n}(t)= \begin{cases}\frac{1}{\sqrt{\pi}} \sin \left(\frac{n}{2} t\right) \mathrm{e}^{-1 / t} t^{-3 / 2} & \text { if } t>0 \\ 0 & \text { if } t \leq 0 .\end{cases}
$$

Let us remark that $y_{n} \in C^{\infty}(\mathbb{R})$ and $y_{n} \in H^{m}(\mathbb{R})$ for any nonnegative integer $m$. Then we consider the following boundary value problem. Let $U=U_{n j p}(x, t)$ be a classical solution to

$$
\begin{cases}\partial_{t} U-\Delta U=0 & \text { in } \Omega \times(0,+\infty), \\ U(x, t)=0 & (x, t) \in \Omega \times(-\infty, 0], \\ U=\Psi_{n j p} & \text { on } A \times \mathbb{R} .\end{cases}
$$

The following decay estimate of exponential type will be crucial. 
Proposition 5.5 For any $n, j$, p, let $U_{n j p}$ solve (5.22). Let us fix $\rho_{0}, 0<\rho_{0}<$ 1. Then there exist positive constants $K_{1}$ and $k_{1}$, depending on $\rho_{0}$ only, such that

$$
\left\|U_{n j p}\right\|_{L^{2}\left(B_{\rho_{0}} \times \mathbb{R}\right)} \leq K_{1} \exp \left(-k_{1} \sqrt{n+j}\right) .
$$

The proof of Proposition 5.5 is rather technical and therefore we postpone it to the end of the section. As a corollary of Proposition 5.5, we obtain the following result.

Lemma 5.6 For any $n, j, p$, and any $D \in X$, we have

$$
\left\|\left(\tilde{\mathcal{D}}_{1}(D)-\tilde{\mathcal{D}}_{1}(\emptyset)\right) \tilde{\psi}_{n j p}\right\|_{\tilde{H}^{\prime}} \leq K_{2} \exp \left(-k_{1} \gamma\left(\tilde{\psi}_{n j p}\right)\right)
$$

where $K_{2}$ depends on $m$ and $b$ only.

Proof. By (5.9) and (5.10), and the relation between $\psi_{n j p}$ and $\tilde{\psi}_{n j p},(5.5)$, we obtain that

$$
\left\|\left(\tilde{\mathcal{D}}_{1}(D)-\tilde{\mathcal{D}}_{1}(\emptyset)\right) \tilde{\psi}_{n j p}\right\|_{\tilde{H}^{\prime}} \leq C_{1}\left\|(\tilde{\mathcal{D}}(D)-\tilde{\mathcal{D}}(\emptyset)) \tilde{G} \psi_{n j p}\right\|_{\tilde{H}^{\prime}} .
$$

Let $U_{n j p}$ solve (5.22). Then we have that the restriction of $U_{n j p}$ to the time interval $(0,2 \pi)$ solves $(5.7)$ with $D=\emptyset$ and boundary datum $\tilde{G} \psi_{n j p}$. By (5.11), we infer that there exists a constant $K_{3}$, depending on $m$ and $b$ only, such that

$$
\left\|\left(\tilde{\mathcal{D}}_{1}(D)-\tilde{\mathcal{D}}_{1}(\emptyset)\right) \tilde{\psi}_{n j p}\right\|_{\tilde{H}^{\prime}} \leq K_{3}\left\|U_{n j p}\right\|_{H^{2,1}\left(B_{7 / 8} \times(0,2 \pi)\right)} .
$$

Then the conclusion follows by Proposition 5.5 and a Caccioppoli type inequality of the form

$$
\left\|U_{n j p}\right\|_{H^{2,1}\left(B_{\rho_{1}} \times(0,2 \pi)\right)} \leq K_{4}\left\|U_{n j p}\right\|_{L^{2}\left(B_{\rho_{0}} \times(0,2 \pi)\right)},
$$

where $0<\rho_{1}<\rho_{0}<1$ and $K_{4}$ depends on $\rho_{0}$ and $\rho_{1}$ only. For similar Caccioppoli type inequalities we refer to [13, Chapter III, Section 6].

Now we almost have what is needed to prove Proposition 5.4 and hence conclude the proof of Theorem 2.5.

Proof of Proposition 5.4. We need to introduce the operator which is adjoint to $\tilde{\mathcal{D}}_{1}$. For any $D \in X \cup\{\emptyset\}$, let us define the bounded and linear operator $\tilde{\mathcal{D}}^{*}(D): \tilde{H} \mapsto \tilde{H}_{0}$ such that, for any $\tilde{\phi} \in \tilde{H}$, we have

$$
\tilde{\mathcal{D}}^{*}(D) \tilde{\phi}=\left.\frac{\partial \tilde{v}}{\partial \nu}\right|_{\tilde{\Gamma}}
$$

where $\tilde{v}$ solves

$$
\begin{cases}\partial_{t} \tilde{v}+\Delta \tilde{v}=0 & \text { in } \tilde{Q}(D), \\ \tilde{v}(x, 2 \pi)=0 & x \in \Omega \backslash D, \\ \tilde{v}=0 & \text { on } \tilde{\Gamma}(D), \\ \tilde{v}=\tilde{\phi} & \text { on } \tilde{\Gamma} .\end{cases}
$$

Then we define $\tilde{\mathcal{D}}_{1}^{*}(D): \tilde{H} \mapsto \tilde{H}^{\prime}$ so that

$$
\left\langle\tilde{\mathcal{D}}_{1}^{*}(D) \tilde{\phi}, \tilde{\psi}\right\rangle_{\tilde{H}^{\prime}, \tilde{H}}=\left\langle\tilde{\mathcal{D}}^{*}(D) \tilde{G}^{*} \tilde{\phi}, \tilde{G} \tilde{\psi}\right\rangle_{\tilde{H}^{\prime}, \tilde{H}}, \quad \text { for any } \tilde{\phi}, \tilde{\psi} \in \tilde{H}
$$


Using the weak formulation of (5.7) and (5.25), it is easy to show that the following adjointness property holds true for any $D \in X \cup\{\emptyset\}$

$$
\left\langle\tilde{\mathcal{D}}_{1}(D) \tilde{\psi}, \tilde{\phi}\right\rangle_{\tilde{H}^{\prime}, \tilde{H}}=\left\langle\tilde{\mathcal{D}}_{1}^{*}(D) \tilde{\phi}, \tilde{\psi}\right\rangle_{\tilde{H}^{\prime}, \tilde{H}}, \quad \text { for any } \tilde{\psi}, \tilde{\phi} \in \tilde{H} \text {. }
$$

It is not difficult to show, with a simple change of variable in time, that (5.24) holds true if we replace $\tilde{\mathcal{D}}_{1}$ with $\tilde{\mathcal{D}}_{1}^{*}$. Then, through the adjointness, we have that for any $n, n^{\prime}, j, j^{\prime}, p, p^{\prime}$

$$
\left|\left\langle\left(\tilde{\mathcal{D}}_{1}(D)-\tilde{\mathcal{D}}_{1}(\emptyset)\right) \tilde{\psi}_{n j p}, \tilde{\psi}_{n^{\prime} j^{\prime} p^{\prime}}\right\rangle_{\tilde{H}^{\prime}, \tilde{H}}\right| \leq K_{5} \exp \left(-k_{1} \max \left\{\gamma\left(\tilde{\psi}_{n j p}\right), \gamma\left(\tilde{\psi}_{n^{\prime} j^{\prime} p^{\prime}}\right)\right\}\right),
$$

where $K_{5}$ depends on $m$ and $b$ only.

Then, recalling Proposition 5.2, the properties of the orthonormal basis $\left\{\tilde{\psi}_{n j p}\right\}$ and of $\gamma$, in particular (5.6), and the decay estimate (5.26) above, we notice that we are exactly in the position of applying the abstract instability theorem stated as Theorem 2.1 in [7]. Hence Proposition 5.4 follows.

Proof of Proposition 5.5. We suppose that $U_{n j p}$ can be written as follows

$$
U_{n j p}(x, t)=V_{n j p}(r, t) f_{j p}(\omega), \quad r=\|x\|, \omega=x /\|x\| .
$$

For the time being, let us denote $V=V_{n j p}(r, t), V_{t}=\partial_{t} V$ and $V_{r}=\frac{\partial V}{\partial r}$, $V_{r r}=\frac{\partial^{2} V}{\partial r^{2}}$. We have that $V$ satisfies

$$
\begin{cases}V_{t}-V_{r r}-\frac{N-1}{r} V_{r}+\frac{j(j+N-2)}{r^{2}} V=0 & \text { in }(0,1) \times(0,+\infty), \\ V(r, t)=0 & (r, t) \in(0,1) \times(-\infty, 0], \\ V(1, t)=y_{n}(t) & t \in(0,+\infty), \\ V(0, t)=0\left(V_{r}(0, t)=0\right) & t \in(0,+\infty), j \geq 1(j=0) .\end{cases}
$$

Let $\hat{V}$ be the Fourier transform of $V$ with respect to time, that is

$$
\hat{V}(r, \xi)=\int_{-\infty}^{+\infty} \mathrm{e}^{-\mathrm{i} \xi t} V(r, t) \mathrm{d} t, \quad \xi \in \mathbb{R}, r \in(0,1) .
$$

By using classical tables of integral transforms, see for instance [8], we have that the Fourier transform of $y_{n}$ is, for any $\xi \in \mathbb{R}$,

$$
\begin{aligned}
\hat{y}_{n}(\xi) & =\frac{\mathrm{e}^{-\sqrt{2|\xi-n / 2|}}}{2 \mathrm{i}}(\cos (\sqrt{2|\xi-n / 2|})-\operatorname{sign}(\xi-n / 2) \mathrm{i} \sin (\sqrt{2|\xi-n / 2|})) \\
- & \frac{\mathrm{e}^{-\sqrt{2|\xi+n / 2|}}}{2 \mathrm{i}}(\cos (\sqrt{2|\xi+n / 2|})-\operatorname{sign}(\xi+n / 2) \mathrm{i} \sin (\sqrt{2|\xi+n / 2|})),
\end{aligned}
$$

hence the following estimate holds

$$
\left|\hat{y}_{n}(\xi)\right| \leq \mathrm{e}^{-\sqrt{2|\xi-n / 2|}}+\mathrm{e}^{-\sqrt{2|\xi-(-n / 2)|}}, \quad \text { for any } \xi \in \mathbb{R} .
$$

We have that $\hat{V}$ solves

$$
\begin{cases}\hat{V}_{r r}+\frac{N-1}{r} \hat{V}_{r}-\left(\mathrm{i} \xi+\frac{j(j+N-2)}{r^{2}}\right) \hat{V}=0 & \text { in }(0,1) \times \mathbb{R}, \\ \hat{V}(1, \xi)=\hat{y}_{n}(\xi) & \xi \in \mathbb{R}, \\ \hat{V}(0, \xi)=0\left(\hat{V}_{r}(0, \xi)=0\right) & \xi \in \mathbb{R}, j \geq 1(j=0) .\end{cases}
$$


If we choose $\sigma, \alpha$ and $\nu$ so that $\sigma^{2}=-\mathrm{i} \xi, 1-2 \alpha=N-1$ and $\alpha^{2}-\nu^{2}=$ $-j(j+N-2)$, that is

$$
\sigma=\frac{\sqrt{2|\xi|}}{2}(\mathrm{i}-\operatorname{sign} \xi), \quad \alpha=\frac{2-N}{2}, \quad \nu=\sqrt{\frac{(N-2)^{2}}{4}+j(j+N-2)}
$$

then, using formulas 5.4.11 and 5.4.12 in [14], we infer that for any $\xi \in \mathbb{R}$, any $r \in(0,1)$,

$$
\hat{V}(r, \xi)=r^{\frac{2-N}{2}}\left(a_{1}^{\xi} J_{\nu}(\sigma r)+a_{2}^{\xi} Y_{\nu}(\sigma r)\right),
$$

where $J_{\nu}$ and $Y_{\nu}$ are Bessel functions of order $\nu$ of first and second kind, respectively, and $a_{1}^{\xi}$ and $a_{2}^{\xi}$ are coefficients depending on $\xi$. Concerning Bessel functions we refer mainly to [22] and [14]. We always restrict ourselves to the case in which $\nu \geq 0$, and we recall here the following basic properties. The Bessel functions have the following asymptotic behaviour as $z \rightarrow 0$

$$
\begin{aligned}
& J_{\nu} \sim z^{\nu}, \quad Y_{\nu} \sim(1 / z)^{\nu}, \quad \text { as } z \rightarrow 0, \text { if } \nu>0, \\
& J_{0} \rightarrow 1, \quad Y_{0} \sim \log z / 2, \quad \text { as } z \rightarrow 0,
\end{aligned}
$$

and these formulas hold for the derivative

$$
\frac{\mathrm{d}}{\mathrm{d} z}\left(z^{-\nu} J_{\nu}(z)\right)=-z^{-\nu} J_{\nu+1}(z), \quad \frac{\mathrm{d}}{\mathrm{d} z}\left(z^{-\nu} Y_{\nu}(z)\right)=-z^{-\nu} Y_{\nu+1}(z) .
$$

Then, it is easy to show that the boundary condition at $r=0$ is satisfied if and only if $a_{2}^{\xi}=0$.

For any $r>0$ and any $\xi \neq 0$, we have that $J_{\nu}(\sigma r) \neq 0$, see for instance [14, Theorem 2, p. 127] and [22, Chapter XV]. Therefore, inserting the boundary condition at $r=1$, we obtain that for any $r \in(0,1)$,

$$
\hat{V}(r, \xi)=r^{\frac{2-N}{2}} \frac{J_{\nu}(\sigma r)}{J_{\nu}(\sigma)} \hat{y}_{n}(\xi), \quad \text { for any } \xi \neq 0 .
$$

We wish to estimate the modulus of $\frac{J_{\nu}(\sigma r)}{J_{\nu}(\sigma)}$. We use the following formula, see [22, formula 3 , p. 498],

$$
J_{\nu}(z)=\frac{(z / 2)^{\nu}}{\Gamma(\nu+1)} \prod_{l=1}^{+\infty}\left(1-\frac{z^{2}}{\tau_{\nu, l}^{2}}\right)
$$

where $0<\tau_{\nu, 1}<\tau_{\nu, 2}<\ldots<\tau_{\nu, l}<\ldots$ are the positive zeroes of $J_{\nu}$ and here $\Gamma(\nu+1)$ is the value of the Gamma function in $\nu+1$.

We infer that

$$
\left|\frac{J_{\nu}(\sigma r)}{J_{\nu}(\sigma)}\right|=r^{\nu} \prod_{l=1}^{+\infty}\left(\frac{\tau_{\nu, l}^{4}+r^{4} \xi^{2}}{\tau_{\nu, l}^{4}+\xi^{2}}\right)^{1 / 2}
$$

We have that

$$
\log \prod_{l=1}^{+\infty}\left(\frac{\tau_{\nu, l}^{4}+r^{4} \xi^{2}}{\tau_{\nu, l}^{4}+\xi^{2}}\right)^{1 / 2} \leq-\frac{1}{2}\left(1-r^{4}\right) \xi^{2} \sum_{l=1}^{+\infty} \frac{1}{\tau_{\nu, l}^{4}+\xi^{2}}
$$


In order to evaluate the right hand side of the last formula, we make use of the following properties of the zeroes of the Bessel functions. First, for any $l \in \mathbb{N}$, the function $\nu \mapsto \tau_{\nu, l}$ is increasing, see [22, p. 508], therefore

$$
\sum_{l=1}^{+\infty} \frac{1}{\tau_{\nu, l}^{4}+\xi^{2}} \geq \sum_{l=1}^{+\infty} \frac{1}{\tau_{[\nu]+1, l}^{4}+\xi^{2}}
$$

where $[\nu]$ denotes the integer part of $\nu$. The so-called interlacing-property, [22, p. 479], implies that

$$
\sum_{l=1}^{+\infty} \frac{1}{\tau_{[\nu]+1, l}^{4}+\xi^{2}} \geq \sum_{l=[\nu]+2}^{+\infty} \frac{1}{\tau_{0, l}^{4}+\xi^{2}} .
$$

By Bôcher's Theorem, see [22, p. 494], we obtain that $\tau_{0, l} \leq(2 l-1) \tau_{0,1}$, for any $l \in \mathbb{N}$. Therefore, if we denote $\tau=\tau_{0,1}$, which is a positive absolute constant, we can conclude that

$$
\sum_{l=1}^{+\infty} \frac{1}{\tau_{\nu, l}^{4}+\xi^{2}} \geq \sum_{l=[\nu]+2}^{+\infty} \frac{1}{\tau^{4}(2 l-1)^{4}+\xi^{2}},
$$

which in turn is greater than or equal to

$$
\int_{[\nu]+2}^{+\infty} \frac{1}{\tau^{4}(2 x-1)^{4}+\xi^{2}} \mathrm{~d} x=\frac{1}{2 \tau|\xi|^{3 / 2}} \int_{\frac{(2[\nu]+3) \tau}{\sqrt{|\xi|}}}^{+\infty} \frac{1}{y^{4}+1} \mathrm{~d} y .
$$

If we estimate the last integral, distinguishing whether the first endpoint of the interval of integration is less than 1 or not, we can find a positive absolute constant $K_{6}$ such that

$$
\frac{1}{2} \sum_{l=1}^{+\infty} \frac{1}{\tau_{\nu, l}^{4}+\xi^{2}} \geq K_{6} \min \left\{\frac{1}{|\xi|^{3 / 2}}, \frac{1}{(2[\nu]+3)^{3} \tau^{3}}\right\} .
$$

We continue by noticing that there exists a positive constant $K_{7}$ such that

$$
\frac{1}{(2[\nu]+3)^{3} \tau^{3}} \geq \frac{K_{7}}{(j+1)^{3}}, \quad \text { for any } j \geq 0 .
$$

Thus, recalling (5.33), there exists $K_{8}>0$ such that we have, for any $r \in(0,1)$ and any $\xi \neq 0$, the following crucial estimate

$$
|\hat{V}(r, \xi)| \leq r^{\nu-\frac{N-2}{2}} \exp \left(-K_{8}\left(1-r^{4}\right) \xi^{2} \min \left\{\frac{1}{|\xi|^{3 / 2}}, \frac{1}{(j+1)^{3}}\right\}\right)\left|\hat{y}_{n}(\xi)\right| .
$$

We recall that $\left|\hat{y}_{n}(\xi)\right|$ can be estimated as in (5.28).

Let us now fix $\rho_{0}, 0<\rho_{0}<1$. We wish to estimate

$$
\int_{B_{\rho_{0}} \times(0,+\infty)}|u(x, t)|^{2} \mathrm{~d} x \mathrm{~d} t=\frac{1}{2 \pi} \int_{0}^{\rho_{0}} \int_{-\infty}^{+\infty} r^{N-1}|\hat{V}(r, \xi)|^{2} \mathrm{~d} \xi \mathrm{d} r .
$$

Here we have used the fact that $\left\|f_{j p}\right\|_{L^{2}(A)}=1$ and Plancherel's Theorem. Then, by (5.35) and (5.28) we have

$$
\int_{B_{\rho_{0}} \times(0,+\infty)}|u(x, t)|^{2} \mathrm{~d} x \mathrm{~d} t \leq \frac{1}{2 \pi} \frac{\rho_{0}^{2 \nu+2}}{2 \nu+2} E,
$$


where

$$
E \leq 4 \int_{-\infty}^{+\infty} \exp \left(-2 K_{8}\left(1-\rho_{0}^{4}\right) \xi^{2} \min \left\{\frac{1}{|\xi|^{3 / 2}}, \frac{1}{(j+1)^{3}}\right\}\right) \mathrm{e}^{-2 \sqrt{2|\xi-n / 2|}} \mathrm{d} \xi .
$$

Since $\nu \geq j$ and $\rho_{0}<1$, then

$$
\frac{1}{2 \pi} \frac{\rho_{0}^{2 \nu+2}}{2 \nu+2} \leq \rho_{0}^{2 j} .
$$

We now turn our attention to the term $E$. We distinguish between two cases.

First, if $n / 4 \geq(j+1)^{2}$, we have, setting $K_{9}=K_{8}\left(1-\rho_{0}^{4}\right)$,

$$
E \leq 4 \int_{|\xi| \leq n / 4} \exp (-\sqrt{2 n}) \mathrm{d} \xi+4 \int_{|\xi| \geq n / 4} \exp \left(-K_{9} \sqrt{n}\right) \exp (-2 \sqrt{2|\xi-n / 2|}) \mathrm{d} \xi,
$$

therefore, for a positive absolute constant $K_{10}$,

$$
E \leq 2 n \exp (-\sqrt{2 n})+K_{10} \exp \left(-K_{9} \sqrt{n}\right) .
$$

We conclude that there exist positive constants $K_{11}, K_{12}$, depending on $\rho_{0}$ only, such that

$$
E \leq\left\{\begin{array}{l}
K_{11} \exp \left(-K_{12} \sqrt{n}\right), \quad \text { if } n / 4 \geq(j+1)^{2}, \\
K_{11}, \quad \text { if } n / 4<(j+1)^{2} .
\end{array}\right.
$$

Then, inserting (5.37) and (5.38) into (5.36), a simple computation yields to (5.23) with constants $K_{1}$ and $k_{1}$ depending on $\rho_{0}$ only.

In order to conclude the proof, we have just to check that if we set

$$
U(x, t)=V(\|x\|, t) f_{j p}(x /\|x\|), \quad(x, t) \in \Omega \backslash\{0\} \times \mathbb{R},
$$

where

$$
V(r, t)=\frac{1}{2 \pi} \int_{-\infty}^{+\infty} \mathrm{e}^{\mathrm{i} t \xi \hat{V}}(r, \xi) \mathrm{d} \xi, \quad(r, t) \in(0,1) \times \mathbb{R}
$$

and $\hat{V}$ is defined as in (5.33), then $U$ can be extended to a continuous function on $\bar{\Omega} \times \mathbb{R}$ which satisfies (5.22).

For this purpose, the following estimate will be crucial. For any $\eta \leq 0$ and any $\xi \in \mathbb{R}$, let $\zeta=\xi+\mathrm{i} \eta$ and $\sigma^{2}=-\mathrm{i} \zeta$, with $\arg (\sigma)$ satisfying $\pi / 4 \leq \arg (\sigma) \leq 3 \pi / 4$. Then, if we apply (5.34) again we obtain that

$$
\left|\frac{J_{\nu}(\sigma r)}{J_{\nu}(\sigma)}\right| \leq r^{\nu}, \quad \text { for any } \eta \leq 0, \xi \neq 0, r \in(0,1) .
$$

Since the support of $y_{n}$ is contained in $[0,+\infty),(5.39)$ and the Paley-Wiener theory, see for instance [10, Theorem 7.4.3], imply that, for any $r \in(0,1)$, the support of $V(r, t)$ is contained in $[0,+\infty)$ as well. Finally, the regularity of $y_{n}$, (5.29), that is the equation and the boundary conditions at $r=0$ and $r=1$ satisfied by $\hat{V}$, the asymptotic behaviour of the Bessel functions as $z \rightarrow 0$, and, when $j=0$, formula (5.32), imply that $V$ satisfies (5.27) and, in turn, that the function $U$ defined above is indeed the solution to (5.22). 


\section{References}

[1] G. Alessandrini, E. Beretta, E. Rosset and S. Vessella, Optimal stability for inverse elliptic boundary value problems with unknown boundaries, Ann. Scuola Norm. Sup. Pisa Cl. Sci. 29 (2000), pp. 755-806.

[2] A. Binder, On an inverse problem arising in continuous casting of steel billets, Appl. Anal. 57 (1995), pp. 341-366.

[3] K. Bryan and L. F. Caudill Jr., Stability and reconstruction for an inverse problem for the heat equation, Inverse Problems 14 (1998), pp. 1429-1453.

[4] B. Canuto, E. Rosset and S. Vessella, Quantitative estimates of unique continuation for parabolic equations and inverse initial-boundary value problems with unknown boundaries, Trans. Amer. Math. Soc. 354 (2002), pp. 491-535.

[5] B. Canuto, E. Rosset and S. Vessella, A stability result in the localization of cavities in a thermic conducting medium, ESAIM: COCV 7 (2002), pp. 521565.

[6] R. Chapko, R. Kress and J.-R. Yoon, On the numerical solution of an inverse boundary value problem for the heat equation, Inverse Problems 14 (1998), pp. 853-867.

[7] M. Di Cristo and L. Rondi, Examples of exponential instability for inverse inclusion and scattering problems, Inverse Problems 19 (2003), pp. 685-701.

[8] A. Erdélyi ed., Tables of Integral Transforms, Volume I, McGraw-Hill, New York Toronto London, 1954.

[9] L. Escauriaza and S. Vessella, Optimal three cylinder inequalities for solutions to parabolic equations with Lipschitz leading coefficients, in G. Alessandrini and G. Uhlmann eds., Inverse Problems: Theory and Applications, American Mathematical Society, Providence R.I., 2003, pp. 79-87.

[10] L. Hörmander, The Analysis of Linear Partial Differential Operators, Volume I, Springer-Verlag, Berlin Heidelberg New York, 1983.

[11] V. Isakov, Some inverse problems for the diffusion equation, Inverse Problems 15 (1999), pp. 3-10.

[12] A. N. Kolmogorov and V. M. Tihomirov, $\epsilon$-entropy and $\epsilon$-capacity of sets in functional spaces, Uspehi Mat. Nauk. 14 (1959), pp. 3-86 (Russian). English translation in Amer. Math. Soc. Transl. 17 (1961), pp. 277-364.

[13] O. A. Ladyženskaja, V. A. Solonnikov and N. N. Ural'ceva, Linear and Quasilinear Equations of Parabolic Type, American Mathematical Society, Providence R.I., 1968.

[14] N. N. Lebedev, Special Functions and their Applications, Prentice-Hall, Englewood Cliffs N.J., 1965.

[15] G. M. Lieberman, Second Order Parabolic Differential Equations, World Scientific, Singapore New Jersey London, 1996. 
[16] J. L. Lions and E. Magenes, Non-Homogeneous Boundary Value Problems and Applications, Volumes I and II, Springer-Verlag, Berlin Heidelberg New York, 1972.

[17] N. Mandache, Exponential instability in an inverse problem for the Schrödinger equation, Inverse Problems 17 (2001), pp. 1435-1444.

[18] A. Morassi and E. Rosset, Stable determination of cavities in elastic bodies, Inverse Problems 20 (2004), pp. 453-480.

[19] C. Müller, Spherical Harmonics, Springer-Verlag, Berlin Heidelberg New York, 1966.

[20] V. Vavilov, E. Grinzato, P. G. Bison, S. Marinetti and M. J. Bales, Surface transient temperature inversion for hidden corrosion characterisation: theory and applications, Int. J. Heat Mass Transfer 39 (1996), pp. 355-371.

[21] S. Vessella, Carleman estimates, optimal three cylinder inequality, and unique continuation properties for solutions to parabolic equations, Comm. PDE 28 (2003), pp. 637-676.

[22] G. N. Watson, A Treatise on the Theory of Bessel Functions, Cambridge University Press, Cambridge, 1944. 